\title{
Botany
}

\section{Réponses adaptatives à un assèchement édaphique chez trois provenances de Chêne liège (Quercus suber L.)}

\begin{tabular}{|r|l|}
\hline Journal: & Botany \\
\hline Manuscript ID & cjb-2020-0214.R3 \\
\hline Manuscript Type: & Article \\
\hline Author: & O1-Jun-2021 \\
\hline Complete List of Authors: & $\begin{array}{l}\text { Abassi, Mejda; Institut National des Recherches en Génie Rurale, Eaux et } \\
\text { Forêts (INRGREF), Laboratoire d'Ecologie Forestière (LR11INRGREF03) } \\
\text { Zouaoui, Refka; Institut National des Recherches en Génie Rurale, Eaux } \\
\text { et Forêts (INRGREF), Laboratoire d'Ecologie Forestière (LR11INRGREF03) } \\
\text { Hachani, Chadlia ; University of Carthage, Faculty of Sciences of Bizerte; } \\
\text { National Institute of Research in Rural Engineering Water and Forests, } \\
\text { Laboratory of Forest Ecology } \\
\text { Béjaoui, Zoubeir; University of Carthage, Faculty of Sciences of Bizerte; } \\
\text { National Institute of Research in Rural Engineering Water and Forests, } \\
\text { Laboratory of Forest Ecology }\end{array}$ \\
\hline Keyword: & $\begin{array}{l}\text { stress hydrique, plasticité phénotypique, variabilité intraspécifique, } \\
\text { micromorphologie foliaire, Boisement }\end{array}$ \\
\hline $\begin{array}{r}\text { Is the invited manuscript for } \\
\text { consideration in a Special } \\
\text { Issue? : }\end{array}$ & Not applicable (regular submission) \\
\hline &
\end{tabular}

\section{SCHOLARONE \\ Manuscripts}




\section{Réponses adaptatives à un assèchement édaphique chez trois provenances de Chêne liège (Quercus suber L.)}

Mejda Abassi, Refka Zouaoui, Chadlia Hachani et Zoubeir Béjaoui

Mejda Abassi : Laboratoire d'Ecologie Forestière (LR11INRGREF03), Institut National de Recherches en Génie Rural, Eaux et Forêts (INRGREF), Université de Carthage, Rue Hédi El karray, El Menzah IV, BP 10, Ariana 2080, Tunisie; e-mail : mej_abassi@yahoo.fr

Refka Zouaoui: Laboratoire d'Ecologie Forestière (LR11INRGREF03), Institut National de Recherches en Génie Rural, Eaux et Forêts (INRGREF), Université de Carthage, Rue Hédi El karray, El Menzah IV, BP 10, Ariana 2080, Tunisie; e-mail :refkazouaoui19@gmail.com

Chadlia Hachani : Faculté des Sciences de Bizerte, Université de Carthage, Jarzouna 7021, Tunisie et Laboratoire d'Ecologie Forestière (LR11INRGREF03), Institut National de Recherches en Génie Rural, Eaux et Forêts (INRGREF), Université de Carthage, Rue Hédi El karray, El Menzah IV, BP 10, Ariana 2080, Tunisie; e-mail : chadliahachanii@gmail.com

Zoubeir Béjaoui: Faculté des Sciences de Bizerte, Université de Carthage, Jarzouna 7021, Tunisie et Laboratoire d'Ecologie Forestière (LR11INRGREF03), Institut National de Recherches en Génie Rural, Eaux et Forêts (INRGREF), Université de Carthage, Rue Hédi El karray, El Menzah IV, BP 10, Ariana 2080, Tunisie; e-mail : zoubeirbe@yahoo.fr

Auteur correspondant: Zoubeir Béjaoui (Faculté des Sciences de Bizerte, Université de Carthage, Jarzouna 7021, Tunisie et Laboratoire d'Ecologie Forestière (LR11INRGREF03), Institut National de Recherches en Génie Rural, Eaux et Forêts (INRGREF), Université de Carthage, Rue Hédi El karray, El Menzah IV, BP 10, Ariana 2080, Tunisie; courrier : zoubeirbe@yahoo.fr 


\section{Résumé}

Les effets du changement climatique induisent dans la région méditerranéenne la recrudescence d'évènements extrêmes comme la sécheresse accentuant la dégradation des écosystèmes forestiers de Quercus suber L. Des jeunes plants de trois provenances tunisiennes de Quercus suber (EF, BM et OZ) ont été évalués pour leur tolérance au manque d'eau en les soumettant durant 90 jours à trois régimes hydriques ( $\mathrm{S} 0$ : irrigation à $100 \%$ de la capacité au champ (CC), S1 : $50 \%$ CC et S2: 25\% CC). De nombreuses variables ont été mesurées (croissance des tiges, surface foliaire, densité et dimension des trichomes et des stomates, échanges gazeux et concentration en chlorophylles). Le déficit hydrique a occasionné une réduction des variables écophysiologiques et une augmentation de la densité des stomates et des trichomes. Une variabilité intraspécifique a été enregistrée. Les plants EF et BM ont montré un indice de plasticité phénotypique supérieur à celui des plants OZ. La meilleure flexibilité vis-à-vis du stress hydrique s'est traduite chez les plants EF par une augmentation de l'EUEi convertie en une croissance plus soutenue des tiges. Dans un contexte global de réchauffement climatique et de fragilité des forêts méditerranéennes, la réussite des nouveaux boisements de Quercus suber exige le choix de provenances les plus adaptées à la faible disponibilité en eau à l'instar de la provenance EF.

Mots clés : Boisement, Quercus suber, stress hydrique, micromorphologie foliaire, variabilité intraspécifique, plasticité phénotypique. 


\section{Introduction}

Les forêts de chêne liège (Quercus suber L.) représentent une composante majeure de l'écosystème méditerranéen occidental et sont reconnues par leur haute valeur écologique, socio-économique et paysagère (Ben Zyane 1998; Bellahirech et al. 2018). Ces forêts notamment celles en Tunisie sont toutefois confrontées à un dépérissement provoqué par les cycles de plus en plus récurrents de sécheresse sous l'effet des changements climatiques, les attaques d'insectes phyllophages, les incendies et la pression anthropique (Sebei et al. 2004). En effet, sur les 150.000 ha de l'aire du chêne, il n'en reste que 73.000 ha dont 45.000 ha sont productifs et 27.500 ha constituent des forêts dégradées (DGF 2010). Une telle situation a été aggravée autant par ces contraintes qui ont limité la régénération naturelle du chêne liège que par l'absence d'opérations sylvicoles de protection et de renouvellement (Hasnaoui 1992 ; Ksontini 1998). Ainsi, les plantations artificielles s'avèrent une solution efficace si les conditions d'élevage en pépinière et les méthodes de plantation seraient maîtrisées. Cette étape passe impérativement par le choix des provenances les mieux adaptées aux contraintes édapho-climatiques du milieu particulièrement le déficit hydrique. En effet, la rareté des ressources en eau constitue le principal facteur limitant pour de nombreuses espèces ligneuses, notamment après leur transplantation. Selon l'IPCC (2014), les effets du changement climatique global devraient augmenter l'intensité et la fréquence des sécheresses dans de nombreuses régions, y compris le bassin méditerranéen (MedECC 2015). La Tunisie fait partie des pays exposés à un déficit hydrique élevé. En effet, les projections climatiques pour la région de la Kroumirie, (Nord-Ouest extrême du pays) abritant la plus grande forêt de chêne liège prévoient à l'horizon 2040-2100, une diminution des précipitations avec un déficit hydrique avoisinant 29 à 69\% (Verner et al. 2018).

Le manque d'eau affecte sensiblement la survie et la croissance des plants de reboisement ou de régénération naturelle, notamment au stade juvénile (Zine El Abidine 2003). En réponse à 
une faible disponibilité en eau, les plantes manifestent le plus souvent des symptômes visibles comme le dessèchement des feuilles, la réduction de l'expansion et de la surface foliaire (Hussain et Ali 2015) et le ralentissement de la croissance caulinaire (Petropoulos et al. 2008). Simultanément, un dysfonctionnement des processus physiologiques fondamentaux en résulte comme la teneur en eau, la conductance mésophyllienne, la conductance des stomates et la synthèse des pigments chlorophylliens (Parkash et Singh 2020). La baisse de la photosynthèse nette est attribuable à une fermeture des stomates, avec pour conséquence une diminution de la conductance à la diffusion du $\mathrm{CO}_{2}$ et/ou à une limitation biochimique du chloroplaste à fixer le $\mathrm{CO}_{2}$ suite à une altération de l'ultrastructure de ces organites (Farquhar et Sharkey 1982 ; Bertolino et al. 2019 ; Parkashet Singh 2020). Afin de s'acclimater au déficit hydrique, les espèces végétales développent certaines stratégies adaptatives d'ordre morphologique et physiologique, souvent associées à une modification des caractères de l'épiderme foliaire (Fraser et al. 2009). Ce dernier joue un rôle clé dans toutes les interactions entre la plante et son environnement et est essentiel pour le maintien de conditions physiologiquement favorables (Glover et al. 2016). En effet ce tissu foliaire est muni de stomates et pour certaines espèces végétales comme le cas de Quercus suber de poils ou trichomes qui assurent un rôle crucial dans l'économie de l'eau (Abdulrahaman 2009). La pubescence des feuilles est un caractère commun aux plantes xériques (Abrams 1994). Cette caractéristique accroit l'efficience de l'utilisation de l'eau en augmentant la résistance de la couche limite de la feuille (Savé et al. 2000). Ces structures épidermiques favorisent également l'augmentation de la réflexion du rayonnement solaire ce qui réduit la température interne de la feuille limitant ainsi la perte de l'eau sous les conditions de déficit hydrique (Bickford 2016). De plus les stomates principal accès aux cellules du mésophylle, jouent un rôle majeur dans le contrôle des échanges gazeux et la régulation efficace des flux d'eau (Hetherington et Woodward 2003 ; Arve et al. 2011).Ces traits sont toutefois tributaires des conditions environnementales 
et peuvent se modifier afin d'atténuer les effets du stress hydrique. En effet, la plasticité phénotypique des traits morphologiques peut contribuer à améliorer les performances de l'espèce végétale et influencer sa survie face aux conditions adverses du milieu (Gratani 2014). La baisse de la surface foliaire figure parmi les traits morphologiques d'adaptation à la faible disponibilité en eau qui permet à la plante de réduire à la fois la transpiration et d'avoir une meilleure gestion de l'eau (Anyia et Herzog 2004). Cette modification est souvent accompagnée par une augmentation du nombre des trichomes et des stomates (Bosu et Wagner 2007 ; Abdulrahaman et Oladele 2011) et de la baisse de l'ouverture des ostioles sous l'effet de la perte de la turgescence des cellules de garde (Bartlett et al. 2016). De telles modifications plastiques permettent aux plantes d'ajuster la surface des pores stomatiques en réponse à la contrainte hydrique assurant l'équilibre entre le besoin de carbone et l'économie de l'eau (Bertolino et al. 2019).

Dans le cadre d'une contribution à la connaissance des conditions favorables à la régénération du chêne liège, l'identification à un stade précoce des provenances les plus adaptées aux conditions de déficit hydrique s'avère incontournable. L'évaluation des mécanismes d'adaptation que développeraient à un stade juvénile les plants de chêne liège autant sur le plan morphologique, micromorphologique que physiologique en réponse à la contrainte hydrique parait nécessaire pour mieux cerner le degré de plasticité phénotypique des provenances testées face à la contrainte hydrique. Cette étape permettra d'identifier à un stade précoce les provenances les mieux adaptées à cette contrainte. Ainsi, l'objectif général du présent travail consiste à caractériser la variabilité intra spécifique du chêne liège en réponse à un stress hydrique modéré ou sévère par la mesure chez des plants de trois provenances de certaines caractéristiques des stomates et des trichomes, la conductance des stomates, la photosynthèse et la concentration en chlorophylles. Par ailleurs, la réduction de la croissance 
autant des tiges que des feuilles représentent les indicateurs clés de la tolérance au stress hydrique chez les provenances testées.

\section{Matériel et méthodes}

\section{Matériel végétal et conditions de culture}

L'expérimentation a porté sur des plants issus de glands de trois populations de chêne liège originaires des forêts naturelles de Ben Metir, Oued Zen et El Feija. Ces trois sites caractéristiques de la région de la Kroumirie (Gouvernorat de Jendouba -Nord-Ouest extrême de la Tunisie), sont sous bioclimat humide supérieur à hiver tempéré et diffèrent par leur conditions géographiques (Figure 1; Tableau 1). Ils ont la particularité de présenter un fort contraste saisonnier entre des hivers pluvieux et une sécheresse estivale très forte estimée par un quotient pluviométrique d'Emberger, Q $<1,5$ (Selmi 1985).

La collecte des glands s'est faite au mois de décembre, de façon aléatoire sur un minimum de 10 pieds-mère par population. Pour cette étude, la population fait référence à l'ensemble des pieds-mère à partir desquels les glands ont été récoltés et le site dont la population est originaire à la provenance. Pour chaque provenance, des glands sains de même poids ont été plantés à la pépinière de l'INRGREF sous un bioclimat semi-aride supérieur à hiver doux $\left(36^{\circ} 50 \mathrm{~N}, 10^{\circ} 14 \mathrm{E}\right.$ et $3 \mathrm{~m}$ d'altitude). La pluviométrie moyenne annuelle est de l'ordre de 475 mm avec des températures moyennes mensuelles minimales de $7,2^{\circ} \mathrm{C}$ et maximales de $34,8^{\circ} \mathrm{C}$. Ces glands ont été disposés dans des pots en plastique de $50 \mathrm{~cm}$ de diamètre et $60 \mathrm{~cm}$ de profondeur, contenant le même poids de substrat $(10 \mathrm{~kg})$ constitué d'un mélange sable terreau $(30 \%-70 \%)$. Au bout de deux années de culture, pour chaque provenance, des plants homogènes de même croissance en hauteur ont été choisis. Ils ont été divisés en trois lots et soumis à trois niveaux de stress hydrique appréciés par la mesure à des paliers décroissants de la capacité au champ (CC): $100 \%$ CC (S0), $50 \%$ CC (S1) et 25\% CC (S2) avec une teneur en eau volumique du sol respective de 33\%, 18\% et 10\%. L'intensité de 
l'irrigation a été quantifiée à l'aide d'un TDR (TDR 300 Soil Moisture Meter type Field Scout Spectrum Technologies). Chaque traitement hydrique a duré 90 jours et a été appliqué du début du mois de juillet à la fin du mois de septembre. A la fin de cette période, plusieurs variables morphologiques, micromorphologiques et physiologiques ont été mesurées. Le dispositif expérimental adopté est en parcelles divisées (Split-Plot) à deux facteurs étudiés (provenances et traitements) avec 4 répétitions complètes (blocs). Les trois provenances de Quercus suber occupent les grandes parcelles (premier étage) et les trois traitements hydriques les petites parcelles (deuxième étage). Chaque unité expérimentale (petite parcelle) est constituée d'une ligne de 4 plants correspondant à un traitement.

Variables mesurées

\section{Croissance des plants}

La croissance des plants des trois provenances de chêne liège sous les trois régimes hydriques a été évaluée par la mesure de la hauteur de la tige principale ( $\mathrm{mm})$, le diamètre au collet $(\mathrm{mm})$, la surface moyenne des feuilles $\left(\mathrm{cm}^{2}\right)$ et l'élongation foliaire $(\mathrm{mm})$. Afin de mieux apprécier l'effet de la contrainte hydrique sur la croissance des plants, le taux de croissance relative (TCR) en hauteur et en diamètre au collet a été calculé en utilisant quatre plants par provenance par bloc et par traitement. Ce paramètre permet d'évaluer l'efficacité de la croissance exprimée par la capacité des plants à produire de la biomasse (Kozlowski et al. 1991). Il est défini par la relation suivante :

$$
T C R n=\frac{\operatorname{Ln} \mathrm{W} 2-\mathrm{Ln} \mathrm{W} 1}{\mathrm{t} 2-\mathrm{t} 1}
$$

Où $\mathrm{W}_{2}$ et $\mathrm{W}_{1}$ représentent respectivement la hauteur ou le diamètre des tiges à la fin et au début d'une durée de stress équivalente à $\mathrm{t}_{2}$ et $\mathrm{t}_{1}$, soit 90 jours pour le cas de cette étude.

L'élongation foliaire a été déterminée par une mesure régulière de la longueur des feuilles nouvellement émises au niveau de l'apex. Ces mesures ont été assurées à l'aide d'un pied à 
coulisse $(0-150 \mathrm{~mm}$, avec une erreur technique de 0,01 mm). Elles ont été suivies du début de l'émergence des nouvelles feuilles jusqu'à l'arrêt de leur croissance et qui correspond à la phase de maturité des feuilles. Elles ont été prises sur 3 feuilles par plant / provenance / bloc/ traitement soit un total de 12 feuilles par provenance. Ces mesures ont permis de déterminer le taux d'élongation foliaire $\left(\mathrm{mm}\right.$. jour $\left.^{-1}\right)$. Il est calculé par la différence entre la mesure de la longueur initiale de la feuille nouvellement émise et celle de sa longueur après un intervalle de temps donné. Pour le cas de notre étude, la vitesse de croissance des feuilles a été suivie à des intervalles réguliers de 3 jours et ce durant 27 jours. En effet cette période correspond au stade d'arrêt de la croissance foliaire et qui coïncide avec la maturité des feuilles de chêne liège. Les divers taux calculés ont permis d'établir la cinétique de la vitesse de la croissance foliaire chez les plants de chêne liège sous les trois traitements hydriques ( $\mathrm{S} 0, \mathrm{~S} 1$ et S2). Par ailleurs, le taux moyen de l'élongation foliaire (TMEF) a été calculé par la différence entre la mesure initiale pour chaque feuille et sa mesure finale au bout de 27 jours de traitement. La surface foliaire a été mesurée à la fin de l'expérimentation sur 60 feuilles par traitement et par provenance ( 5 feuilles par plant x 3 répétitions x 4 blocs) à l'aide d'un logiciel OPTIMAS 6 .

\section{$\underline{\text { Micromorphologie des feuilles }}$}

La micromorphologie des feuilles a été mesurée à l'aide de la technique des empreintes épidermiques. Les observations ont été réalisées grâce à une caméra vidéo type Hitachi KP-D 40 Color Digtal, montée à un microscope de type Leitz Dialux 22E (agrandissement 250 fois) et reliée à un ordinateur. L'image affichée sur l'écran de l'ordinateur est analysée à l'aide d'un logiciel Win Dias. A la fin de l'expérimentation, les mesures ont été réalisées sur des feuilles matures situées au même rang, sur un rameau bien éclairé. Chaque donnée est la moyenne de 16 observations ( 4 observations par feuille et 1 feuille par plant x 4 répétitions/ provenance et par traitement). Les variables mesurées sont: la densité et la dimension des 
stomates, la densité et la dimension des trichomes, la surface relative des pores stomatiques et la surface des trichomes.

La densité des stomates (DS) a été déterminée par le comptage de leur nombre par $\mathrm{mm}^{2}$ de surface foliaire. La dimension des stomates a été appréciée par la mesure en $\mu \mathrm{m}$ de la longueur (a) et de la largeur (b) de l'ouverture de l'ostiole. Ces paramètres ont servi à la détermination de la surface relative des pores stomatiques (SRP) exprimée par la formule décrite par Meinder et Mansfield (1968) :

$$
\operatorname{SRP}(\%)=\left[\frac{(a b)}{4}\right] \cdot D S \cdot \pi \cdot 10^{-4}
$$

La densité des trichomes (DT) a été évaluée par le comptage du nombre de chaque empreinte par $\mathrm{mm}^{2}$ d'épiderme alors que leur largeur (LT) a été déterminée par la mesure en $\mu \mathrm{m}$ de l'empreinte de la base de chaque trichome.

La surface ou couverture des trichomes (ST) a été calculée par la formule décrite par Dilcher (1974) :

$$
S T\left(m m^{2}\right)=(L T / 2)^{2} \times \pi \times D T
$$

\section{$\underline{\text { Echanges gazeux }}$}

Le taux de photosynthèse nette (A) et la conductance stomatique (gs) ont été déterminés automatiquement à l'aide d'un analyseur portable à infrarouge (IRGA, LCA model 4, Analytical Company Ltd. Hoddesdon, Hertfordshire), à raison de quatre plants choisis aléatoirement par provenance par bloc et par traitement. Les mesures ont été effectuées entre 8 heures et 10 heures, sur des feuilles entières de rang 5 à 8 les plus exposées au rayonnement solaire. Les mesures ont été prises après 90 jours d'exposition aux traitements hydriques sous les conditions ambiantes suivantes : une surface foliaire dans la pince, $\mathrm{S}=6,25 \mathrm{~cm}^{2}$, une humidité relative $(\mathrm{RH})$ variant de $40 \%$ à $50 \%$, un flux de photons dans la pince au niveau de la feuille variant de 800 à $900 \mu \mathrm{mol} \mathrm{m} \mathrm{m}^{-2} \cdot \mathrm{s}^{-1}$, la température de la feuille a varié de 30 à $32^{\circ} \mathrm{C}$ et 
la teneur en $\mathrm{CO}_{2}$ a été de $350 \mu \mathrm{mol} . \mathrm{mol}^{-1}$. L'efficience d'utilisation de l'eau intrinsèque (EUEi) a été calculée en divisant la photosynthèse nette par la conductance stomatique (Farquhar et Sharkey 1982).

\section{$\underline{\text { Dosages des pigments chlorophylliens }}$}

Les concentrations en pigments chlorophylliens ont été évaluées à la fin des traitements et ce sur les mêmes feuilles ayant servi à la mesure des échanges gazeux. L'extraction des pigments chlorophylliens (Arnon 1967) a porté sur des disques foliaires d'une masse fraiche de trois grammes. La densité optique est mesurée à $663 \mathrm{~nm}$ pour la chlorophylle (a) et à 645nm pour la chlorophylle (b). Les concentrations en chlorophylles (a) et (b), exprimées en mg. $\mathrm{g}^{-1} \mathrm{MF}$ sont calculées par les formules suivantes :

$$
\begin{aligned}
& \text { Chl (a) }(\mathrm{mmol} / \mathrm{mgMF})=[12.7 \mathrm{DO}(663)-2,69(645)] * 0.2 \\
& \text { Chl }(\mathrm{b})(\mathrm{mmol} / \mathrm{mgMF})=[22,9 \mathrm{DO}(645)-4,68(663)] * 0.2 \\
& \mathrm{Chl}(\mathrm{a}+\mathrm{b})=[20,2 \mathrm{DO}(645)+8,02 \mathrm{DO}(663)] * 0.2
\end{aligned}
$$

\section{Indice de plasticité phénotypique}

Un indice de plasticité phénotypique a été calculé afin d'apprécier les traits d'adaptation que pourraient développer les trois provenances en réponse au stress hydrique (Valladares et al. 2000). Cet indice qui varie de zéro à un, a été calculé pour chaque variable considérée et provenance comme la différence entre les valeurs moyennes minimales et maximales parmi les trois traitements hydriques divisée par la valeur moyenne maximale en utilisant quatre plants par provenance par bloc et par traitement.

$$
\text { Indice de plasticité phénotypique }=(\max -\min ) / \max
$$


Pour chaque provenance, une plasticité phénotypique moyenne a été déterminée en calculant la moyenne des indices de plasticité obtenus pour chacune des variables mesurées.

\section{Analyse statistique}

Les données collectées des différentes variables considérées dans cette expérimentation ont été soumises à une analyse de la variance à trois critères ou facteurs de classification selon un modèle en Split-Plot. Les deux facteurs provenance et traitement sont considérés comme fixes (effet fixe) et le facteur bloc ou répétition est considéré comme aléatoire (effet aléatoire). La comparaison des moyennes des différentes modalités de chacun des deux facteurs étudiés a été effectuée par le test de Newman et Keuls (Dagnelie 2011). Toutes les analyses statistiques ont été réalisées à l'aide de la procédure GLM de SAS/STAT (SAS 2004).

\section{Résultats}

\section{Effet du stress hydrique sur la croissance des jeunes plants de chêne liège}

Les interactions (traitement et provenance), ainsi que les effets du traitement hydrique et de la provenance, ont affecté de manière significative plusieurs variables morpho-physiologiques (Tableau 2). Sous témoin, les plants BM se sont démarqués par le taux de croissance relative en hauteur des tiges (TCRh) le plus élevé $2,17 \mathrm{~mm} \cdot \mathrm{j}^{-1}$, contre $1,39 \mathrm{~mm} \cdot \mathrm{j}^{-1}$ et 1,26 mm.j. respectivement chez les plants $\mathrm{EF}$ et $\mathrm{OZ}$. Le stress hydrique induit par l'irrigation à $50 \% \mathrm{CC}$ et $25 \% \mathrm{CC}$ a réduit $(\mathrm{p}<0,05)$ le TCRh des plants de Quercus suber mais de manière plus importante chez les plants OZ (fig. 2). Ces derniers ont été les plus sévèrement affectés notamment sous le seuil $25 \% \mathrm{CC}$ avec une réduction de l'ordre de $57 \%$ du témoin contre $32 \%$ et $27 \%$ respectivement chez les plants $\mathrm{BM}$ et $\mathrm{EF}$ (fig. 2). L'accroissement relatif radial (TCRd) a été également freiné sous l'effet de ce seuil hydrique sévère (25\% CC). Les plants EF ont enregistré le plus faible taux de réduction soit $14 \%$ du témoin contre $26 \%$ et $33 \%$ respectivement pour BM et OZ. Les valeurs de la surface moyenne des feuilles (SMF) enregistrées pour les plants témoins ont été comparables chez les provenances étudiées et ont 
été de l'ordre de $6,43 \mathrm{~cm}^{2}$ (fig. 2). La réponse des plants de chêne liège à la contrainte hydrique a été exprimée par une baisse significative $(\mathrm{p}<0,05)$ de la SMF par rapport aux plants témoins. Sous $50 \%$ CC, les réductions enregistrées ont été de $21 \%, 22 \%$ et $30 \%$ respectivement chez les plants EF, OZ et BM. Sous le stress plus sévère S2 (25\% CC), la SMF a été davantage réduite. Les plants EF montrent le plus faible taux de réduction soit 39\% du témoin contre $47 \%$ et $49 \%$ respectivement chez les plants BM et OZ. La vitesse de l'élongation des feuilles témoins ou traitées exprimée par le taux de l'élongation foliaire (TEF) a atteint son maximum au bout de 15 jours pour les plants BM et $\mathrm{OZ}$ et 18 jours pour ceux EF. Cette phase de croissance foliaire accrue a été suivie par un ralentissement progressif du TEF jusqu'à arrêt complet (fig. 3). L'arrêt de l'élongation foliaire traduit la maturité des feuilles et qui a été atteint au bout de 27 jours pour le cas des plants de chêne liège testés. Sous l'effet du stress hydrique, un ralentissement du TEF a été enregistré chez tous les plants de chêne liège. Ce ralentissement a été tributaire de la provenance considérée et d'autant plus important que le niveau de stress hydrique a été plus élevé. Sous le seuil 25\% $\mathrm{CC}$, nous avons enregistré au bout de 15 jours, une baisse respective par rapport au témoin de la vitesse de l'élongation foliaire de l'ordre de $72 \%$ et $52 \%$ chez BM et OZ et de $48 \%$ chez EF au bout de 18 jours. D'autre part, sous l'effet du stress hydrique, un raccourcissement de la phase de maturité des feuilles a été enregistré chez les plants OZ. Ces plants ont atteint leur maturité foliaire au bout de 24 jours de traitement sous S1 et S2, soit une précocité de 3 jours par rapport au témoin (S0). Par ailleurs, le taux moyen de l'élongation foliaire (TMEF, mm.j${ }^{1}$ ) exprimant la vitesse de la croissance foliaire durant la période de traitement a été retardé sous l'effet des deux niveaux de stress hydrique appliqués. Sous 50\% CC, les trois populations ont développé un TMEF comparable variant de 0,46 à $0,48 \mathrm{~mm} . \mathrm{j}^{-1}$. Sous le niveau hydrique plus sévère $(25 \% \mathrm{CC})$, les plants EF montrent le plus fort TMEF $\left(0,33 \mathrm{~mm} \cdot \mathrm{j}^{-1}\right)$ suivis par les plants OZ $\left(0,279 \mathrm{~mm} \cdot \mathrm{j}^{-1}\right)$ et BM $\left(0,204 \mathrm{~mm} \cdot \mathrm{j}^{-1}\right)$. 
Effet du stress hydrique sur les caractéristiques des stomates et des trichomes

L'observation microscopique des empreintes épidermiques a montré la présence de stomates et de poils en forme d'étoile sur la face inférieure des feuilles de Quercus suber (fig. 4). Les résultats de l'analyse de la variance ont montré que les effets des traitements, de la provenance ou leur interaction ont été significatifs pour les variables DS, LPS, DT, LT et ST (Tableau 2). Sous les conditions optimales (S0 :100\% CC), les plants BM et EF ont montré des caractéristiques stomatiques similaires avec une densité qui a varié de 646 à $727 \mathrm{st} / \mathrm{mm}^{2}$ et une ouverture en longueur des ostioles de 19 à $20 \mu \mathrm{m}$ (Tableau 3). A l'opposé, les plants OZ se sont différenciés des plants BM et EF par un plus faible nombre de stomates $\left(516 \mathrm{st} / \mathrm{mm}^{2}\right)$ mais ces derniers sont dotés d'une plus grande ouverture en longueur des pores stomatiques $(22 \mu \mathrm{m})$. De plus, ces plants ont montré le plus grand nombre de trichomes $\left(74 \mathrm{Tr} / \mathrm{mm}^{2}\right)$ contre $\left(48 \mathrm{Tr} / \mathrm{mm}^{2}\right)$ chez les plants $\mathrm{BM}$ et $\mathrm{EF}$. Le niveau hydrique $25 \% \mathrm{CC}$, a modifié de manière significative la densité des trichomes et des stomates, l'ouverture en longueur des ostioles et la largeur des trichomes. Ceci s'est traduit par une augmentation du nombre de stomates de l'ordre de $32 \%$ chez les plants BM et EF contre une variation non significative chez les plants OZ (Tableau 3). En parallèle, l'ouverture en longueur des ostioles a été réduite $(\mathrm{p}<0,05)$ chez tous les plants de chêne liège sous l'effet d'une fermeture partielle des stomates en réponse au déficit hydrique. Toutefois, une variabilité entre les trois provenances a été dégagée. En effet, les plants EF et BM se sont distingués par une plus forte réduction de l'ouverture en longueur des ostioles qui a été respectivement de l'ordre de $33 \%$ et $31 \%$ contre $21 \%$ chez les plants OZ. Le nombre de trichomes a fortement augmenté chez les plants de Quercus suber sous l'effet du seuil hydrique $(25 \% \mathrm{CC})$. La densité de ces structures épidermiques a été 3,6 à 3,8 fois plus élevée que celle du témoin chez les plants EF et BM contre 1,8 fois chez les plants OZ. La largeur des trichomes a également augmenté $(\mathrm{p}<0,05)$ supposant un épaississement de ces structures sous l'effet du stress hydrique. Le plus fort taux 
d'accroissement (34\% du témoin) a été enregistré chez les plants BM et EF, contre $20 \%$ chez les plants OZ. La modification de ces caractéristiques s'est traduite par une augmentation de la surface des trichomes ou couverture en trichomes de l'ordre de $87 \%$ chez les plants BM et EF et de $65 \%$ chez OZ. Par ailleurs pour les trois provenances, les ratios densité des stomates /surface foliaire (DS/SF) et densité des trichomes / surface foliaire (DT/SF) ont été d'autant plus élevés que le stress hydrique a été plus sévère (Tableau 3). Les plants BM se sont différenciés $(p<0,05)$ des plants EF et $\mathrm{OZ}$ par la valeur moyenne la plus élevée du ratio DS/SF. L'augmentation de ces ratios indique que la baisse de la surface foliaire chez les plants de Quercus suber a été accompagnée par une augmentation à la fois du nombre de stomates et de trichomes.

\section{Effet du stress hydrique sur les échanges gazeux et la concentration en chlorophylles}

Le stress hydrique a réduit significativement $(\mathrm{p}<0,05)$ la conductance stomatique $(\mathrm{gs})$ et le taux d'assimilation nette (A) chez les plants de chêne liège. Cette baisse a été tributaire du niveau de stress appliqué et de la provenance en question (fig. 6). Sous S1 (50\% CC), la conductance stomatique a baissé de $42 \%$ chez les plants BM et de $30 \%$ à $32 \%$ chez EF et OZ. En parallèle, on assiste à une réduction de l'assimilation nette de $24 \%, 33 \%$ et $15 \%$ respectivement chez les plants $\mathrm{BM}$, EF et OZ. Sous $25 \% \mathrm{CC}$, ces variables ont été sévèrement réduites. Ceci s'est traduit par une réduction de $71 \%$ de (A) et $79 \%$ de (gs) chez les plants OZ et de l'ordre de $67 \%$ pour ces deux variables chez les plants BM. A l'opposé, les plants EF, ont montré un comportement différent qui a été illustré par une forte baisse de gs $(64 \%$ du témoin) contre une réduction de moindre importance pour A (44\%). La baisse plus importante de gs par comparaison à $\mathrm{A}$, a induit chez les plants EF une augmentation significative de l'efficience intrinsèque de l'utilisation de l'eau (EUEi) qui pourrait traduire chez cette provenance un meilleur comportement vis-à-vis de ce seuil de stress hydrique. Par ailleurs, une corrélation positive hautement significative $(\mathrm{r}=0,81, \mathrm{p}<0,01)$ a été enregistrée entre le 
ratio trichomes / stomates (DT/DS) et l'efficience intrinsèque de l'utilisation de l'eau chez les plants EF. Cette relation souligne le rôle important de ces structures épidermiques dans le contrôle de la perte de l'eau sous des conditions hydriques déficientes.

La contrainte hydrique a affecté négativement $(\mathrm{p}<0,05)$ la synthèse de la chlorophylle totale chl $(a+b)$ chez les plants de chêne liège (fig. 6). Sous $25 \% \mathrm{CC}$, cette variable a baissé significativement chez tous les plants. Toutefois les plants BM et EF ont montré une meilleure aptitude à tolérer ce niveau de stress hydrique à travers une réduction plus faible de la concentration en chlorophylle totale de l'ordre de $38 \%$ du témoin contre $73 \%$ chez OZ.

\section{Indice de plasticité phénotypique}

Une plasticité phénotypique différente entre les trois provenances en réponse au stress hydrique induit par l'irrigation à $25 \% \mathrm{CC}$ a été mise en évidence. Cette plasticité a été exprimée par les traits micromorphologiques de l'épiderme foliaire (DS, LPS, DT, LT et ST) contrairement aux variables morphologiques (TCRh, TCRd et SF) qui ont montré une faible variation entre provenances (Tableau 2). Ainsi, l'indice moyen de plasticité phénotypique calculé sous $25 \%$ CC a montré que les plants BM et EF ont développé le plus fort indice qui est de l'ordre de 0,52 contre 0,34 pour les plants OZ (fig. 5). Ceci traduit chez ces deux provenances une plus grande plasticité phénotypique adaptative leur permettant de mieux exprimer ces traits épidermiques foliaires afin de s'adapter aux conditions contraignantes du déficit hydrique.

\section{Discussion}

Variation des traits morpho-physiologiques des provenances de chêne liège en réponse au stress hydrique

Cette étude a permis de dégager une variabilité morphologique entre les trois provenances de Quercus suber qui peut refléter une adaptation à la diversité des conditions environnementales de leurs habitats d'origine. Cette variabilité serait reliée à des caractères phénotypiques qui 
dépendent principalement de facteurs génétiques et environnementaux comme cela a été prouvé chez plusieurs populations de chêne liège natives de différentes régions de la Tunisie (Abdessamad et al. 2014). Cette différenciation entre les populations en réponse aux différentes conditions de l'environnement souligne la forte variabilité intra spécifique que présente le genre Quercus (Batos et al. 2017). Les résultats ont montré une différence dans le comportement morpho-physiologique des trois provenances de chêne liège en réponse au stress hydrique. Ceci souligne la variabilité intra spécifique que présente cette espèce face aux contraintes abiotiques du milieu (Ksontini et al. 1998 ; Abassi et al. 2019). Par ailleurs, l'analyse des données sous les deux niveaux de stress hydrique appliqués durant 90 jours a permis de considérer le traitement à $50 \%$ de la capacité au champ comme modéré dans la mesure où les réductions relatives des variables morphologiques mesurées par rapport à leurs témoins respectifs ont été inférieures à 50\% (Atmane et al. 2005). Néanmoins, le traitement sous $25 \% \mathrm{CC}$ a constitué un seuil de discrimination entre les provenances testées. En effet, sous ce seuil hydrique sévère, le TCR des tiges en hauteur et au diamètre au collet, le taux d'élongation des feuilles ainsi que la surface moyenne des feuilles ont été significativement réduits chez tous les plants de chêne liège. De telles modifications morphologiques sont considérées comme une réponse précoce au déficit hydrique qui permet aux plantes de réduire leur transpiration et augmenter ainsi l'efficacité de l'utilisation de l'eau (Xu et Zhou 2005). En outre, le ralentissement de la croissance en hauteur engendrant un rabougrissement des plants de chêne liège serait une réponse adaptative qui permettrait de raccourcir le trajet hydraulique chez la plante et contribuer ainsi à abaisser le potentiel hydrique (Woodruff et al. 2004). Ces mécanismes sont cruciaux pour la survie des arbres notamment dans l'environnement méditerranéen connu par son aridité saisonnière engendrée par une nonpluviosité durant la période estivale (Leroux 2001). Sous le niveau hydrique sévère $(25 \%$ $\mathrm{CC})$, les plants EF enregistrent le plus faible taux de réduction du TCRh comparativement aux 
plants $\mathrm{BM}$ et $\mathrm{OZ}$. Cette capacité à maintenir un taux de croissance plus important durant le stress hydrique pourrait être considérée chez cette provenance comme un indicateur de tolérance à la sécheresse (Tschaplinski et al. 2006). Par ailleurs, les plants de Quercus suber se sont caractérisés par des feuilles à surface foliaire réduite qui est un trait d'adaptation aux conditions sèches du milieu (Ksontini et al. 1998). En effet, les espèces du genre Quercus caractéristiques de l'écosystème méditerranéen se distinguent par des feuilles épaisses et de petite taille (Simões et al. 2020). Cette spécificité leur conférerait un potentiel photosynthétique plus élevé via l'augmentation de la conductance mésophyllienne entre les espaces intercellulaires foliaires et les sites de carboxylation stimulant ainsi la capacité photosynthétique (Pena-Rojas et al. 2005). Le ralentissement du taux d'élongation foliaire enregistré chez les plants de chêne liège sous l'effet du stress hydrique s'est traduit par une réduction de leur surface moyenne foliaire. Ces transformations seraient sous l'effet de la baisse du potentiel hydrique des cellules qui aurait conduit à une réduction de la pression de turgescence, moteur de la croissance cellulaire. Les cellules sont alors plus petites, ce qui se traduit par des feuilles de taille plus réduite avec une plus faible capacité à intercepter la lumière induisant un potentiel photosynthétique plus bas et par effet retour une croissance plus ralentie. Ainsi, plus la surface foliaire est importante mieux est la croissance (Xu et Zhou 2008). Dans ce contexte, les plants EF ont développé sous l'effet du stress hydrique (25\% CC) le taux d'élongation foliaire le plus rapide, une surface moyenne des feuilles plus élevée ainsi que le plus fort taux d'assimilation nette comparativement aux plants BM et notamment OZ. Ces résultats suggèrent chez les plants de cette provenance une meilleure plasticité vis-àvis du stress hydrique leur conférant une plus forte aptitude à tolérer la faible disponibilité en eau, contrairement aux plants $\mathrm{OZ}$ qui ont été plus susceptibles aux effets du stress hydrique. Néanmoins, ces derniers ont développé une stratégie d'esquive à cette contrainte hydrique à travers une précocité de la phase de maturité de leurs feuilles. Cette sorte d'adaptation permet 
aux plantes de s'harmoniser à l'environnement de production, en échappant partiellement ou complètement au stress hydrique (Levitt 1980).

Adaptations micro morphologiques foliaires et échanges gazeux

L'observation des empreintes épidermiques a montré que la face inférieure des feuilles de Quercus suber est munie de nombreux stomates et de trichomes en forme d'étoile qui est une caractéristique des Quercus périméditerranéens (Safou et Saint-Martin 1989). Ces structures en plus de la cuticule constituent les caractères épidermiques foliaires qui permettent au chêne liège, espèce sclérophylle, de résister à la sècheresse (Simões et al. 2020). Les plants de Quercus suber ont développé une densité stomatique moyenne de $630 \mathrm{st} / \mathrm{mm}^{2}$ qui est plus élevée que celle enregistrée chez des chênes des régions tempérées humides des forêts de l'Europe occidentale comme le cas de Quercus petraea (333 st $/ \mathrm{mm}^{2}$ ) (Yucedag et al. 2019) ou Quercus robur (260 st $/ \mathrm{mm}^{2}$ ) (Batos et al. 2010). Par contre, cette densité est plus faible que celle développée par des formations de Quercus suber des écosystèmes fragiles des espaces méditerranéens (867 à 1037 st $/ \mathrm{mm}^{2}$ ) (Souidi et al. 2014). Nos résultats ont fait également ressortir une variabilité phénotypique dans la densité des trichomes et des stomates chez les trois provenances de chêne liège. Les plants BM et EF ont montré un nombre de trichomes et de stomates comparable avec des ostioles de même ouverture en longueur. A l'opposé les plants $\mathrm{OZ}$ se sont différenciés par une densité en trichomes 1,3 fois plus élevée contre une densité stomatique 1,5 fois plus faible que celle des plants EF et BM. Les trois provenances présentant un gradient altitudinal et latitudinal différent, cette variation phénotypique en termes de densité des stomates ou des trichomes peut être attribuée à des facteurs génétiques ou refléter une adaptation aux conditions éco-géographiques des sites d'origine (Bertolino et al. 2019). Selon Wang et al. (2007), la densité des stomates est fortement contrôlée par les facteurs génétiques alors que leur flexibilité est régulée par les conditions environnementales changeantes (Wang et al. 2007). En outre, les travaux de George et al. (2019), ont montré 
chez plusieurs provenances de Quercus robur L. présentant un gradient différent de précipitation et de sécheresse que la variation des traits fonctionnels entre les provenances est en partie reliée aux régimes de sécheresse relatifs à leurs sites d'origine. L'effet du stress hydrique sévère $(25 \% \mathrm{CC})$ a enclenché une série de modifications dans la structure de l'épiderme foliaire des plants de chêne liège. La densité et la couverture en trichomes ainsi que la densité des stomates et l'ouverture en longueur des ostioles ont été significativement affectées par le stress hydrique contrairement à la largeur des ostioles. Ces résultats montrent une faible plasticité de l'ouverture en largeur des ostioles des plants de Quercus suber en réponse au stress hydrique.

Le déficit hydrique provoqué par l'irrigation à 25\% CC des plants de Quercus suber a induit un épaississement des trichomes et une augmentation de leur densité qui serait reliée pour ce dernier trait à la baisse de la surface moyenne des feuilles comme en atteste l'augmentation du ratio DT/SMF. Il s'en est résulté une couverture foliaire en trichomes plus abondante qui a été 7,7 fois plus élevée que celle du témoin chez les plants BM et EF contre 2,9 fois chez les plants OZ. Partant de ces considérations, nous pouvons avancer que les plants EF et BM seraient mieux protégés contre la perte d'eau que les plants OZ. En effet, l'augmentation de la couverture en trichomes serait attribuée au besoin de la plante à ralentir sa transpiration afin de contribuer efficacement à améliorer sa résistance au stress hydrique (Ning et al. 2016). Ces structures épidermiques constitueraient un modèle adaptatif plastique à la sècheresse à travers leur capacité à limiter la perte de l'eau en augmentant la résistante de la couche limite à l'interface feuille - atmosphère (Meng et al. 2014). Ces transformations à l'échelle des trichomes ont été accompagnées par une augmentation de la densité des stomates concomitante à une réduction de l'ouverture en longueur du pore stomatique. Pareilles modifications plastiques des traits stomatiques permettent à ces provenances d'ajuster la surface des pores stomatiques en réponse aux contraintes environnementales afin de réguler le 
flux des échanges gazeux (Bertolino et al. 2019). Les modifications de ces structures épidermiques sont considérées en plus d'autres caractères morphologiques ou anatomique comme la taille réduite des feuilles, l'épaississement de la feuille et de la cuticule et la réduction de la dimension des stomates (Simões et al. 2020) comme des traits d'adaptation que développe le chêne liège pour résister à la faible disponibilité en eau et aux épisodes de sécheresses de plus en plus récurrents sous l'effet du changement climatique (IPCC 2014). Par ailleurs, l'augmentation du nombre de stomates serait reliée à la réduction de la surface moyenne des feuilles des plants de chêne liège sous l'effet du stress hydrique (Brunel et Binet 1967 ; Li et al. 2017) comme en témoigne l'augmentation enregistrée du ratio DS/SMF. Leur fermeture serait provoquée par la perte de la turgescence des cellules de garde entourant l'ostiole (Outlaw 2003). Ce mécanisme est considéré comme une adaptation des plantes à la sécheresse vu le rôle fondamental des stomates dans la régulation de la transpiration aussi bien à travers leur nombre qu'à leur aptitude à se fermer en réponse au stress perçu exerçant ainsi un contrôle étroit de la perte de l'eau (Barbieri et al. 2012). De plus, la fermeture des stomates est le principal mécanisme des espèces forestières méditerranéennes à feuilles persistantes comme le cas de Quercus suber pour réguler la perte d'eau par la transpiration et éviter les dommages causés par la déshydratation des cellules et des tissus (MartínezVilalta et al. 2002). Pareilles modifications des structures épidermiques foliaires sont considérées comme des ajustements phénotypiques développés par les plants de Quercus suber afin de s'adapter aux conditions de déficit hydrique induit par une irrigation durant 90 jours à $25 \%$ CC. Nos résultats ont mis en évidence une variabilité de l'intensité de ces réponses entre les provenances testées. Les plants EF et BM ont développé une plasticité phénotypique des traits micromorphologiques foliaires supérieure à celle des plants OZ. Cette meilleure plasticité a été reflétée par la capacité de ces deux provenances à produire différents phénotypes en réponse au stress hydrique (Settele et al. 2014). Ceci s'est traduit par une plus 
forte couverture en trichomes, une densité stomatique plus importante et une fermeture plus élevée des ostioles qui sont des traits plastiques permettant une réponse instantanée des plantes aux changements de leur environnement (Nicotra et al. 2010). La fermeture partielle des stomates a réduit significativement la conductance stomatique (gs) et a induit une baisse du taux d'assimilation nette (A) chez les plants de Quercus suber reflétant ainsi une limitation stomatique de la photosynthèse. Les plants EF ont pu maintenir sous l'effet du déficit hydrique sévère $(25 \% \mathrm{CC})$ un taux photosynthétique plus élevé que celui développé par les plants $\mathrm{BM}$ et notamment $\mathrm{OZ}$ traduisant une meilleure tolérance à la sécheresse chez ces plants. Les espèces les plus tolérantes à la sécheresse limitent la fermeture des stomates de manière à permettre une assimilation substantielle de carbone en situation de contrainte, ce qui leur permet d'améliorer leur efficience d'utilisation de l'eau. Ceci a été le cas des plants EF qui ont enregistré une augmentation de l'efficience de l'utilisation intrinsèque de l'eau sous l'effet du stress hydrique. Cette aptitude réduirait chez ces plants la quantité d'eau perdue par molécule de $\mathrm{CO}_{2}$ assimilée (Farooq et al. 2009). D'autre part, les traits morphologiques foliaires incluant la densité des stomates et des trichomes peuvent affecter les échanges gazeux et leur relation avec les principaux facteurs environnementaux comme le stress hydrique (Xu et Zhou 2008). Nos résultats ont montré une corrélation positive entre le ratio trichome / stomates et l'efficience de l'utilisation intrinsèque de l'eau (EUEi) chez les plants EF. Cette relation indiquerait chez ces plants un rôle important autant des stomates que des trichomes dans la tolérance au déficit hydrique comme cela a été prouvé chez Solanum lycopersicum (tomate) (Galdon-Armero et al. 2018). Ces auteurs voient dans ce ratio un outil prometteur pour la sélection de nouvelles variétés cultivées avec une meilleure efficacité de l'utilisation de l'eau. En parallèle à la baisse de l'assimilation nette, la synthèse de la chlorophylle a été également perturbée chez les plants des trois provenances de Quercus suber induisant une réduction de la concentration en chlorophylle totale $(a+b)$. Cet effet serait 
attribué aux dommages causés aux chloroplastes par les espèces réactives de l'oxygène (ERO) sous l'effet du stress hydrique (Smirnoff 1995). En outre, la réduction de la synthèse chlorophyllienne indiquerait une inhibition de la photosynthèse sous l'effet d'une limitation non stomatique. Par concordance aux travaux de Farquhar et Sharkey (1982), ces résultats indiqueraient que la photosynthèse des plants de Quercus suber aurait été limitée aussi bien par des facteurs stomatiques que non stomatiques. Toutefois, d'autres hypothèses stipulent que les effets stomatiques prédominent sous stress hydrique modéré alors que sous stress sévère ce sont plutôt les limitations biochimiques qui prévalent (Flexas et al. 2009). Nos résultats ont montré que sous le stress modéré $(50 \% \mathrm{CC})$, la synthèse de la chlorophylle totale a été faiblement perturbée chez les plants BM et EF, mais l'a été de manière significative chez les plants OZ. Par contre, sous le seuil sévère $25 \%$ CC ce pigment chlorophyllien a baissé significativement chez les plants des trois provenances. A la lumière de ces résultats, nous pouvons avancer pour le cas des plants BM et EF que la photosynthèse aurait été limitée par des limitations stomatiques sous l'effet du stress hydrique modéré (50\% CC) auxquelles se seraient ajoutées celles biochimiques sous le stress hydrique sévère $(25 \% \mathrm{CC})$. Pour les plants OZ, sous les deux niveaux hydriques se sont autant les limitations stomatiques que métaboliques qui auraient inhibé la photosynthèse.

\section{Conclusion}

Les trois provenances de Quercus suber ont montré une faible variation des traits de croissance comparativement à ceux relatifs à la micro-morphologie des feuilles. Ces provenances ont montré une capacité à exprimer différents phénotypes des traits micromorphologiques foliaires en réponse à la faible disponibilité en eau induite par le niveau hydrique sévère sous $25 \%$ de la capacité au champ. Cependant cette plasticité phénotypique a été tributaire de la provenance dans la mesure où les plants EF et BM ont montré un indice moyen de plasticité supérieur à celui des plants OZ. Cette flexibilité des traits épidermiques 
s'est traduite notamment chez les plants de la provenance EF par le maintien d'un niveau plus élevé de l'assimilation nette et d'une efficience de l'utilisation intrinsèque de l'eau convertie en une croissance plus soutenue des tiges en hauteur ou au diamètre au collet. Cette étude ouvre la voie à l'évaluation de la plasticité phénotypique des espèces forestières dans leur milieu naturel afin d'identifier les individus ou génotypes les plus adaptés aux conditions contraignantes du milieu. Cette initiative permettrait de préserver les écosystèmes méditerranéens de plus en plus fragilisés par les effets des changements climatiques à travers leur reforestation. L'utilisation des traits micromorphologiques foliaires comme proxy pour la détermination de la plasticité phénotypique en parallèle aux mesures écophysiologiques permettrait de mieux comprendre les mécanismes d'adaptation que développeraient les provenances ou génotypes face aux contraintes abiotiques du milieu.

\section{Bibliographie}

Abassi, M., Lamhamedi, M. S., Hachani, C., et Béjaoui, Z. 2019.Variabilité interspécifique et réponses morphophysiologiques à la salinité des principales espèces méditerranéennes de Salix. Can. J. For. Res. 49: 606-615. doi.org/10.1139/cjfr-2018-0491.

Abdessamad, A., Dhib, I., Baraket, Gh, Ksontini, M. et Salhi-Hannachi, A. 2014. Evaluation of Phenotypic Diversity by Use of Variable Analysis Multi of Various Populations of Oak Cork (Quercussuber L.) in Tunisia. Open Journal of Ecology. 4: 861-872. http://dx.doi.org/10.4236/oje.2014.414072.

Abdulrahaman, A. A. 2009. Morphological and epidermal adaptations to water stress in some ornamental plant species. Thesis, University of Ilorin, Ilorin, Nigeria.

Abdulrahaman, A. A. et Oladele, F. A. 2011. Response of trichomes to water stress in two species of Jatropha. Insight Bot. 1:15-21. doi: 10.5567/BOTANY-IK.2011.15.21. 
Anyia, A.O. et Herzog, H. 2004. Water-use efficiency, leaf area and leaf gas exchange of cowpeas under mid-season drought. Eur. J. Agron. 20: 327339.http://dx.doi.org/10.1016/S1161-0301(03)00038-8.

Arnon, A. N. 1967. Method of extraction of chlorophyll in the plants. Agron. J. 23: 112-121.

Arve, L. E., Torre, S., Olsen, J. E. et Tanino, K. K. 2011. Stomatal responses to drought stress and air humidity.Dans Abiotic stress in plants-Mechanisms and adaptations. Edité par A. Shanker. Intech Open. ISBN: 978-953-307-394-1.

Atmane, R., Lemsellek, J., Bousarhal, A., et Abdellatif, R. 2005. Citrus roots tocks response to salinity. Biotechnol. Agron. Soc. Environ. 9(1): 65-73.

Abrams, M. D. 1994. Genotypic and phenotypic variation as stress adaptations in temperate tree species: a review of several case studies. Tree Physiol. 14: 833-842.

Barbieri, G., Vallone, S., Orsini, F., Paradiso, R., De Pascale, S., Negre-Zakharov, F., et Maggio, A. 2012.Stomatal density and metabolic determinants mediate salt stress adaptation and water use efficiency in basil (Ocimumbasilicum L.). J. Plant Physiol. 169(17): 1737-1746. doi:10.1016/j.jplph.2012.07.001. PMID: 22840325.

Bartlett, M. K., Klein, T., Jansen, S., Choat, B., et Sack, L. 2016. The correlations and sequence of plant stomatal, hydraulic, and wilting responses to drought. Proc. Nat. Acad. Sci. 113(46): 13098-13103. doi: 10.1073/pnas.1604088113.

Batos, B., Vilotić, D., Orlović, S. et Miljković, D. 2010. Inter and intra-population variation of leaf stomatal traits of Quercus robur 1. In northern Serbia. Arch. Biol. Sci., Belgrade. 62(4): 1125-1136. DOI: 10.2298/ABS1004125B 1125.

Batos, B., Miljković, D., Perović, M., et Orlović, S. 2017. Morphological variability of Quercus robur L. leaf in Serbia. Genetika. 49(2): 529-541. DOI: 10.2298/GENSR1702529B. 
Bellahirech, A., Inácio, M. L., Ben Jamâa, M. L., et Nóbrega, F. 2018. Ophiostomatoid fungi associated with the ambrosia beetle Platypus cylindrus in cork oak forests in Tunisia. Tunis. J. Plant Prot. 13: 61-76.

Ben Zyane, M., 1998. La subéraie marocaine, produit économique et social à développer. Dans Actes du Séminaire Méditerranéen sur la Régénération des Forêts du Chêneliège, Tabarka, Tunisie, 22-24 Octobre 1996. Ann. INRGREF. № spécial. pp. 12-21.

Bertolino, L. T., Caine, R. S., et Gray, J. E. 2019. Impact of stomatal density and morphology on water-use efficiency in a changing world. Front. Plant Sci.10: 1-11.

Bickford, C. P. 2016. Ecophysiology of leaf trichomes. Funct. Plant Biol. 43(9): 807-814.

Bosu, P. P., et Wagner, M. R. 2007. Effects of induced water stress on leaf trichome density and foliar nutrients of three elm (Ulmus) species: implications for resistance to the Elm Leaf Beetle. Environ. Entomol. 36: 595-601.

Brunel, J. P et Binet, P. 1967. Physiologie végétale II. Edité par Editions Doin.

Dagnelie, P. 2011. Statistique théorique et appliquée Tome 2. Inférence statistique à une et à deux dimensions. Bruxelles, Belgique : De Boeck.

Dilcher, D. L. 1974. Approach to the identification of angiosperms leaf remains. Bot. Rev. 40: $1-157$.

Direction Générale des Forêts (DGF). 2010. Inventaire des forêts par télédétection - Résultat du deuxième inventaire forestier et pastoral national. Ministère de la Défense Nationale, Ministère de l'Agriculture, des Ressources Hydrauliques et de la Pêche et Ministère de l'Enseignement Supérieur et de la Recherche Scientifique. 195 p.

Farooq, M., Wahid, A., Kobayashi, N., Fujita, D., et Basra, S. 2009. Plant drought stress: Effects, mechanisms and management. Dans Sustainable Agriculture. Springer: Dordrecht, the Netherlands. pp. 153-188. 
Farquhar, G. D. et Sharkey, T. D. 1982. Stomatal conductance and photosynthesis. Annu. Rev. Plant Physiol. 33: 317-345.

Flexas, J., Baron, M., Bota, J., Ducruet, J.M., Gallé, A., Galmés, J., Jiménez, M., Pou, A., Ribas-Carbo, M., Sajnani, C., Tomàs, M., et Medrano, H. 2009. Photosynthesis limitations during water stress acclimation and recovery in the drought-adapted Vitis hybrid Richter-110 (V. berlandieri x V.rupestris). J. Exp. Bot. 60: 2361-2377.

Fraser, L. H., Greenall, A., Carlyle, C., Turkington, R. et Friedman, C. R. 2009. Adaptive phenotypic plasticity of Pseudoroegneria spicata: response of stomatal density, leaf area and biomass to changes in water supply and increased temperature. Ann. Bot. 103: 769-775.

Galdon-Armero, J., Fullana-Pericas, M., Mulet,P.A., Conesa, M.A., Martin, C. et Galmes, J. 2018. The ratio of trichomes to stomatais associated with water use efficiency in Solanum lycopersicum (tomato). Plant J. 96 (3): 607-619. doi: 10.1111/tpj.14055.

George J. P., Theroux-Rancourt, G., Rungwattana, K., Scheffknecht, S., Momirovic, N., Neuhauser, L., Weißenbacher, L., Watzinger, A.etHietz, P. 2019. Assessing adaptive and plastic responses in growth and functional traits in a 10-year-old common garden experiment with pedunculate oak (Quercus robur L.) suggests that directional selection can drive climatic adaptation. Evol. Appl. 13(9): 2422-2438. doi: 10.1111/eva.13034.

Glover, B. J., Airoldi, C. A., et Moyroud, E. 2016. Epidermis: Outer cell layer of the plant. eLS. 1-7. doi: 10.1002/9780470015902.a0002072.pub3

Gratani, L. 2014. Plant phenotypic plasticity in response to environmental factors. Adv. Bot. 2014. https://doi.org/10.1155/2014/208747.

Hasnaoui, B. 1992. Chênaies du Nord de la Tunisie: Ecologie et régénération. Thèse, Université de Provence, Marseille, France. 
Hetherington, A. M., et Woodward, F. I. 2003. The Role of stomata in sensing and driving environmental change. Nature. 424: 901-908.

Hussain, B., et Ali, B. 2015. Leaf longevity in plants under water stress. Indian. J. Plant Sci. 4(4): 127-133.

Intergovernmental Panel on Climate Change (IPCC) 2014. Climate Change 2014: Synthesis Report. Contribution of Working Groups I, II and III to the Fifth Assessment Report of the Intergovernmental Panel on Climate Change. Geneva, Switzerland, pp. 151.

Kozlowski, T. T., Kramer, P. J., et Pallardy, S. G. 1991. The physiological ecology of woody plants. Academic Press, San Diego, California, USA. pp. 657. ISBN-10: 0124241603.

Ksontini, M., Louguet, P., Laffray, D., Rejeb, M. N. 1998. Comparaison des effets de la contrainte hydrique sur la croissance, la conductance stomatique et la photosynthèse de jeunes plants de chênes méditerranéens (Quercus suber, Q. faginea, Q. coccifera) en Tunisie. Anal. Sci. Forest. 55 (4): 477-495.

Leroux, M. 2001. Les climats subtropicaux dits «méditerranéens» et les climats de la Méditerranée (1re partie). L'information géographique. 65(4): 304-320.

Levitt J. 1980. Responses of plants to environmental stresses. Vol. II, Water, radiation, salt, and other stresses. Academic Press, New York, USA. pp. 3-211.

Li, Y., Li, H., Li, Y. et Zhang, S. 2017. Improving water-use efficiency by decreasing stomatal conductance and transpiration rate to maintain higher ear photosynthetic rate in drought-resistant wheat. Crop J. 5(3): 231-239.

Martínez-Vilalta, J., Prat, E., Oliveras, I., Piñol, J. 2002. Xylem hydraulic properties of roots and stems of nine Mediterranean woody species. Oecologia. 133: 19-29.

MedECC 2015. Risks associated to climate and environmental changes in the Mediterranean region [en ligne]. Disponible sur http://www.medecc.org/ [consulté le 3 Septembre 2020]. 
Meinder, H. et Mansfield, T. A. 1968. Physiology of Stomata. McGraw Hill, New York, USA.

Meng, F., Peng, M., Pang, H., et Huang, F. 2014. Comparison of photosynthesis and leaf ultrastructure on two black locust (Robinia pseudoacacia L.). Biochem. Syst. Ecol. 55: 170-175.

Nicotra, A. B., Atkin, O. K., Bonser, S. P., Davidson, A. M., Finnegan, E. J., Mathesius, U., Poot, P., Purugganan, M. D., Richards, C. L., Valladares, F. et van Kleunen, M. 2010. Plant phenotypic plasticity in a changing climate. Trends Plant Sci. 15(12): 684-92. doi: 10.1016/j.tplants.2010.09.008.

Ning, P., Wang, J., Zhou, Y., Gao, L., Wang, J., et Gong, G. 2016. Adaptional evolution of trichome in Caragana korshinskii to natural drought stress on the loess plateau, China. Ecol. Evol. 6(11): 3786-3795.

Outlaw, Jr. W. H. 2003. Integration of cellular and physiological functions of guard cells. Crit. Rev. Plant Sci. 22(6): 503-529.

Parkash, V. et Singh, S. 2020. A review on potential plant-based water stress indicators for vegetable crops. Sustainability. 12: 3945. doi:10.3390/su12103945.

Pena-Rojas, K. Aranda, X. Joffre, R. Fleck, I. 2005. Leaf morphology, photochemistry and water status changes in resprouting Quercus ilex during drought. Funct Plant Biol. 32:117-130

Petropoulos, S. A., Daferera, D., Polissiou, M. G., et Passam, H. C. 2008. The effect of water deficit stress on the growth, yield and composition of essential oils of parsley. Sci. Hortic. 115(4): 393-397.

Safou, O. et Saint-Martin, M. 1989. Le trichome foliaire de quelques Quercus périméditerranéens. Bull. Soc. Bot. France. 136(4-5): 291-304. DOI: 10.1080/01811797.1989.10824854. 
SAS Institute Inc. 2004.SAS/STAT ® 9.1 User’s Guide. Cary, NC, USA: SAS Institute Inc.

Savé, R., Biel, C., de Herralde, F. 2000. Leaf pubescence, water relations and chlorophyll fuorescence in two subspecies of Lotus Creticus L. Biol. Plant. 43 : 239-244.

Sebei, H., Albouchi, A., Rapp, M., et El Aouni, M. H. 2004. Productivité en biomasse du chêne liège dans une séquence de dégradation de la suberaie à Cytise de Kroumirie (Tunisie). Ann. For. Sci. 61: 347-361. DOI: 10.1051/forest:2004028.

Selmi, M. 1985. Différenciation des sols et fonctionnement des écosystèmes forestiers sur grès numidien de Kroumerie (Tunisie). Thèse de doctorat en sciences naturelles, Université de Nancy1, CNRS, France.

Settele, J., Scholes, R., Betts, R., Bunn, S., Leadley, P., Nepstad, D., Overpeck, T. J. et Taboada, M. A. 2014. Terrestrial and inland water systems. Dans Climate change 2014 impacts, adaptation, and vulnerability. Part A: global and sectoral aspects. Contribution of working group II to the fifth assessment report of the Intergovernmental Panel on Climate Change. Edité par C. B. Field, V. R. Barros, D. J. Dokken, K. J. Mach, M. D. Mastrandrea, T.E. Bilir, M. Chatterjee, K. L. Ebi, Y. O. Estrada, R. C. Genova, B. Girma, E. S. Kissel, A. N. Levy, S. MacCracken, P. R. Mastrandrea, and L. L. White. Cambridge University Press, Cambridge, United Kingdom. pp. 271-359.

Simões, R., Rodrigues, A., Ferreira-Dias, S., Miranda, I. et Pereira, H. 2020. Chemical Composition of Cuticular Waxes and Pigments and Morphology of Leaves of Quercussuber Trees of Different Provenance. Plants. 9(1165): 1-15. doi:10.3390/plants9091165.

Smirnoff, N. 1995. Antioxidant systems and plant response to the environment. Dans Environment and Plant Metabolism: Flexibility and Acclimation. Edité par V. Smirnoff. BIOS Scientific Publishers, Oxford, UK. 
Souidi, Z., Larbi, H., Hamimed, A., et Kattar, S. 2014. Adaptation des formations de chêneliège (Quercus suber) de l'Algérie occidentale tellienne face aux changements climatiques. Dans Actes du Séminaire : Integrated Protection in Oak Forests, Isle Sur la Sorgue, Avignon, France, 07 - 11 Octobre, 2013. Edité par C. Villemant et P. A. Ruiu. IOBC-WPRS Bull. 101: 1-9.

Tschaplinski, T. J., Abraham, P. E., Jawdy, S. S., Gunter, L. E., Martin, M. Z., Engle, N. L., Yang, X., et Tuskan, G. A. 2006. The nature of the progression of drought stress drives differential metabolomic responses in Populus deltoides. Ann. Bot. 124(4): 617-626. doi: 10.1093/aob/mcz002.

Valladares, F., Wright, S. J., Lasso, E., Kitajima, K.et Pearcy, R. W. 2000. Plastic phenotypic response to light of 16 congeneric shrubs from a Panamanian rainforest. Ecology. 81(7): 1925-1936. doi.org/10.1890/0012-9658.

Verner, D., Treguer, D., Redwood, J., Christensen, J., McDonnell, R., Elbert, C. et Konishi, Y. 2018. Climate Variability, Drought, and Drought Management in Tunisia's Agricultural Sector. World Bank.

Wang, Y., Chen, X. et Xiang, Ch. B. 2007. Stomatal density and bio-water saving. J.I.P.B. 49 (10): 1435-1444.https://doi.org/10.1111/j.1672-9072.2007.00554.x.

Woodruff, D. R., Bond, B. J., et Meinzer, F. C., 2004. Does turgor limit growth in tall trees? Plant, Cell. Environ. 27: 229-236.

Xu, Z. Z.et Zhou G. S., 2005. Effects of water stress and nocturnal temperature on carbon allocation in the perennial grass Leymus chinensis. Physiologia Plantarum. 123: 272280.

$\mathrm{Xu}, \mathrm{Z}$. Z. et Zhou, G. S., 2008. Responses of leaf stomatal density to water status and its relationship with photosynthesis in a grass. J. Exp. Bot. 59(12): 3317-3325. 
Yücedağ, C., Sanders, J.,Musah, M., et Gailing, O. 2019. Stomatal density in Quercus petraea and Q. robur natural populations in Northern Turkey. Dendrobiology. 81: 5864. doi: 10.12657/denbio.081.007.

Zine el Abidine, A., 2003. Le dépérissement des forêts au Maroc: analyse des causes et stratégie de lutte. Sécheresse. 14(4) : 209-218. 


\section{Liste des tableaux}

Tableau 1. Caractéristiques géographiques et climatiques des sites des trois provenances de Quercus suber : Ben Metir, El Feija et Oued Zen.

Tableau 2. Valeurs et signification du test F (Fischer-Snédécor) issues de l'analyse de la variance selon un modèle en Split-Plot des différentes variables mesurées

Tableau 3. Variabilité morphologique intra-spécifique de la surface foliaire et des caractéristiques stomatiques et des trichomes entre les provenances de Quercus suber (Ben Metir (BM), El Feija (EF) et Oued Zen (OZ)). Valeurs moyennes plus ou moins ( \pm ) l'écarttype des combinaisons Provenances x Traitements et comparaison multiple des moyennes des provenances et des traitements par le test de Newman-Keuls. 


\section{Liste des figures}

Fig. 1. Localisation des provenances de chêne liège dans la région de la Kroumirie (Nord Ouest de la Tunisie).

Fig. 2. Taux de croissance relative en hauteur (TCRh) (A), du diamètre au collet (TCRd) (B) et surface foliaire (C) chez trois provenances de Quercus suber : Ben Metir (BM), El Feija $(\mathrm{EF})$ et Oued Zen $(\mathrm{OZ})$ soumis à trois traitements hydrique : témoin $(100 \% \mathrm{CC}, \mathrm{S} 0), 50 \% \mathrm{CC}$ (S1) et 25\% CC (S2). Pour chaque provenance, les moyennes suivies de la même lettre ne sont pas significativement différents selon le test de Newman et Keuls $(\mathrm{p}<0,05)$. ${ }^{*}$ Taux de réduction du TCRh, TCRd et SF enregistré chez les trois provenances sous $25 \% \mathrm{CC}$.

Fig. 3. Taux d'élongation foliaire chez les plants des trois provenances de Quercus suber: Ben Metir (A), El Feija (B) et Oued Zen (C) soumis à trois traitements hydrique : témoin $(100 \% \mathrm{CC}, \mathrm{S} 0), 50 \% \mathrm{CC}(\mathrm{S} 1)$ et $25 \% \mathrm{CC}(\mathrm{S} 2), \mathrm{n}=12$ (moyenne $\pm \mathrm{SD})$

Fig. 4. Observation microscopique des empreintes épidermiques foliaires sur la face abaxiale des feuilles de trois provenances de Quercus suber: Ben Metir (BM), El Feija (EF) et Oued Zen $(\mathrm{OZ})$ soumis à trois traitements hydrique : témoins $(\mathrm{S} 0)$, soumis à $50 \% \mathrm{CC}(\mathrm{S} 1)$ et soumis à $25 \% \mathrm{CC}(\mathrm{S} 2)$ durant 90 jours. L'échelle représente $50 \mu \mathrm{m}$.

Fig. 5. Classification des trois provenances de Quercus suber (Ben Metir (BM), El Feija (EF) et Oued Zen $(\mathrm{OZ})$ ) selon l'indice moyen de plasticité phénotypique calculé pour les quatre variables micromorphologiques (densité des stomates, longueur du pore stomatique, densité des trichomes, largeur du trichome et surface relative des trichomes). Les lettres différentes indiquent une différence significative à $\mathrm{p}<0,05$ selon le test de Newman et Keuls entre les provenances.

Fig. 6. Effet du stress hydrique sur la conductance stomatique (A), l'assimilation nette (B), l'efficience d'utilisation de l'eau intrinsèque $(C)$ et la concentration en chlorophylle $(a+b)(D)$ chez trois provenances de Quercus suber: Ben Metir (BM), El Feija (EF) et Oued Zen (OZ) 
soumis à trois traitements hydrique : témoin $(100 \% \mathrm{CC}, \mathrm{S} 0), 50 \% \mathrm{CC}(\mathrm{S} 1)$ et $25 \% \mathrm{CC}(\mathrm{S} 2)$. Des lettres différentes au-dessus des barres indiquent une différence significative à $\mathrm{p}<0,05$ selon le test de Newman et Keuls entre les provenances de chêne liège et les traitements hydriques 
Tableau 1: Caractéristiques géographiques et climatiques des sites d'origine des trois populations de Quercus suber : Ben Metir (BM), El Feija (EF) et Oued Zen (OZ).

\begin{tabular}{lccc}
\hline Provenance & Ben Metir & El Feija & Oued Zen \\
\hline Longitude & $32.47^{\circ} 48^{\prime} 36^{\prime \prime} \mathrm{E}$ & $32.43^{\circ} 84^{\prime} 22^{\prime \prime} \mathrm{E}$ & $32.48^{\circ} 22^{\prime} 49^{\prime \prime} \mathrm{E}$ \\
Latitude & $40.65^{\circ} 89^{\prime} 8^{\prime \prime} \mathrm{N}$ & $40.40^{\circ} 26^{\prime} 7^{\prime \prime} \mathrm{N}$ & $40.75^{\circ} 63^{\prime} 0^{\prime \prime} \mathrm{N}$ \\
Altitude (m) & 800 & 780 & 675 \\
Température moyenne $\left({ }^{\circ} \mathbf{C}\right)$ & 15 & 14,3 & 14,9 \\
Pluviométrie $(\mathbf{m m})$ & 1500 & 1100 & 1200 \\
\hline
\end{tabular}


Tableau 2 : Valeurs et signification du test F (Fischer-Snédécor) issues de l'analyse de la variance selon un modèle en Split-Plot des différentes variables mesurées.

\begin{tabular}{|c|c|c|c|}
\hline \multirow{2}{*}{ Variables } & \multicolumn{3}{|c|}{$\begin{array}{l}\text { Valeurs du test } \mathrm{F} \text { de Fisher-Snédécor } \\
\text { (F observé) }\end{array}$} \\
\hline & $\begin{array}{l}\text { Provenances } \\
\text { (P) }\end{array}$ & $\begin{array}{l}\text { Traitements } \\
\text { (T) }\end{array}$ & $\begin{array}{l}\text { Interaction } \\
(\mathrm{P} \times \mathrm{T})\end{array}$ \\
\hline TCR h & $3,68^{*}$ & $29,52^{* * *}$ & $15,9^{* *}$ \\
\hline TCRd & 2,51 & $70,58^{* * *}$ & $4,45^{* *}$ \\
\hline TEF & 0,4 & $5,84^{* *}$ & 1,70 \\
\hline SMF & 1,26 & $62,35^{* * *}$ & 0,69 \\
\hline DS & $88,63^{* * *}$ & $76,89^{* * *}$ & $6,28^{* *}$ \\
\hline LPS & $19,03^{* *}$ & $236,17^{* * *}$ & $7,41^{* * *}$ \\
\hline 1PS & 1,6 & 2,5 & 0,7 \\
\hline SRP & 0,21 & 0,94 & 0,32 \\
\hline DT & 0,58 & $211,58^{* * *}$ & $13,26^{* * *}$ \\
\hline $\mathrm{LT}$ & $8,17^{*}$ & $228,56^{* * *}$ & $10,14^{* * *}$ \\
\hline $\mathrm{ST}$ & $4,9^{*}$ & $353,2^{* * *}$ & $21,4^{* *}$ \\
\hline $\mathrm{DS} / \mathrm{SMF}$ & $9,74 * *$ & $94,45 * * *$ & $4,07^{* *}$ \\
\hline $\mathrm{DT} / \mathrm{SMF}$ & 1,70 & $148^{* * *}$ & $5,06^{* *}$ \\
\hline A & $6,99^{*}$ & $286,16^{* * *}$ & $15,80^{* *}$ \\
\hline gs & 1,29 & $1061,8^{* * *}$ & $5,23^{* *}$ \\
\hline EUEi & $5,90^{* *}$ & $5,84^{* *}$ & $7,63^{* * *}$ \\
\hline $\operatorname{Chl}(a+b)$ & 1,5 & $5,90^{* *}$ & 0,64 \\
\hline \multicolumn{4}{|c|}{$\begin{array}{l}\text { : effet significatif pour } \alpha=0,05 ; * * \text { : effet hautement significatif pour } \alpha=0,01 ; * * * \text { : effet très } \\
\text { lautement significatif pour } \alpha=0,001 ; \text { TCRh : Taux de croissance relative en hauteur ; TCRd : Taux de } \\
\text { roissance relative au diamètre au collet ; TEF : Taux d'élongation foliaire ; SMF : Surface moyenne des } \\
\text { euilles; DS : Densité stomatique ; LPS : Longueur du pore stomatique ; IPS : largeur du pore stomatique ; } \\
\text { RP : Surface relative des pores stomatiques ; DT : Densité des trichomes ; LT : Largeur à la base du } \\
\text { richome ; ST : Surface des trichomes ; DS/SMF : Densité des stomates / Surface moyenne des feuilles; } \\
\text { OT/SMF :Densité des trichomes / Surface moyenne des feuilles; A : Assimilation nette ; gs : Conductance } \\
\text { tomatique ; EUEi : Efficience de l'utilisation de l'eau intrinsèque ; Chl }(\mathrm{a}+\mathrm{b}): \text { Chlorophylle }(\mathrm{a}+\mathrm{b})\end{array}$} \\
\hline
\end{tabular}


Tableau 3 : Variabilité morphologique intra-spécifique des caractéristiques stomatiques et des trichomes entre les provenances de Quercus suber (Ben Metir (BM), El Feija (EF) et Oued Zen (OZ)). Valeurs moyennes plus ou moins $( \pm$ ) l'écart-type des combinaisons Provenances $\mathrm{x}$ Traitements et comparaison multiple des moyennes des provenances et des traitements par le test de Newman-Keuls

\begin{tabular}{|c|c|c|c|c|c|}
\hline \multirow[b]{2}{*}{ Variable } & \multirow[b]{2}{*}{ Traitement } & \multicolumn{3}{|c|}{ Provenance } & \multirow{2}{*}{$\begin{array}{l}\text { Moyenne } \\
\text { traitement }\end{array}$} \\
\hline & & BM & $\mathbf{E F}$ & $\overline{\mathrm{OZ}}$ & \\
\hline Densité des & S0 & $727 \pm 59$ & $646 \pm 96$ & $517 \pm 76$ & $630 \mathrm{c}$ \\
\hline stomates & S1 & $778 \pm 108$ & $698 \pm 107$ & $609 \pm 170$ & $695 \mathrm{~b}$ \\
\hline$\left(\mathrm{mm}^{2} /\right.$ surface & S2 & $1070 \pm 141$ & $974 \pm 105$ & $664 \pm 94$ & $903 \mathrm{a}$ \\
\hline foliaire) & $\begin{array}{l}\text { Moyenne } \\
\text { provenance }\end{array}$ & $858,33 \mathrm{a}$ & $772,65 \mathrm{~b}$ & $596,54 \mathrm{c}$ & \\
\hline Longueur du & S0 & $20,26 \pm 0,76$ & $19,04 \pm 0,94$ & $22,15 \pm 1,07$ & $20,48 \mathrm{a}$ \\
\hline pore & S1 & $18,06 \pm 1,90$ & $17,43 \pm 1,06$ & $18,88 \pm 1,20$ & $18,12 \mathrm{~b}$ \\
\hline stomatique & S2 & $13,98 \pm 1,36$ & $12,76 \pm 1,28$ & $17,50 \pm 1,3$ & $14,75 \mathrm{c}$ \\
\hline$(\mu \mathrm{m})$ & $\begin{array}{l}\text { Moyenne } \\
\text { provenance }\end{array}$ & $17,43 \mathrm{~b}$ & $16,41 \mathrm{~b}$ & $19,51 \mathrm{a}$ & \\
\hline Densité des & S0 & $49 \pm 13$ & $49 \pm 8$ & $74 \pm 6$ & $57,56 \mathrm{c}$ \\
\hline trichomes & S1 & $71 \pm 24$ & $58 \pm 4$ & $92 \pm 17$ & $73,85 \mathrm{~b}$ \\
\hline$\left(\mathrm{mm}^{2} /\right.$ surface & S2 & $191 \pm 43$ & $178 \pm 52$ & $134 \pm 40$ & $167,81 \mathrm{a}$ \\
\hline foliaire) & $\begin{array}{l}\text { Moyenne } \\
\text { provenance }\end{array}$ & $103,75 \mathrm{a}$ & $95,35 \mathrm{a}$ & $100,12 \mathrm{a}$ & \\
\hline Largeur du & S0 & $18,31 \pm 2,49$ & $17,93 \pm 1,91$ & $18,25 \pm 1,43$ & $18,17 \mathrm{c}$ \\
\hline \multirow[t]{3}{*}{ trichome $(\mu \mathrm{m})$} & S1 & $22,5 \pm 2,30$ & $22,18 \pm 1.86$ & $19,31 \pm 1,07$ & $21,33 \mathrm{~b}$ \\
\hline & $\mathrm{S} 2$ & $27,87 \pm 1,70$ & $26,68 \pm 1,88$ & $22,75 \pm 1,91$ & $25,77 \mathrm{a}$ \\
\hline & $\begin{array}{l}\text { Moyenne } \\
\text { provenance }\end{array}$ & $22,89 \mathrm{a}$ & $22,27 \mathrm{a}$ & $20,10 \mathrm{~b}$ & \\
\hline Surface des & S0 & $1,47 \pm 0,85$ & $1,26 \pm 0,69$ & $1,94 \pm 0,18$ & $1,56 \mathrm{c}$ \\
\hline trichomes & S1 & $2,81 \pm 0,76$ & $2,26 \pm 0,78$ & $2,66 \pm 0,82$ & $2,58 \mathrm{~b}$ \\
\hline \multirow[t]{2}{*}{$\left(\mathrm{mm}^{2}\right)$} & S2 & $11,43 \pm 1,66$ & $9,82 \pm 1,36$ & $5,41 \pm 0,95$ & $8,87 \mathrm{a}$ \\
\hline & $\begin{array}{l}\text { Moyenne } \\
\text { provenance }\end{array}$ & $5,24 \mathrm{a}$ & $4,45 \mathrm{a}$ & $3,34 \mathrm{~b}$ & \\
\hline \multirow[t]{4}{*}{$\mathrm{DS} / \mathrm{SF}$} & S0 & $1,24 \pm 0,34$ & $1,12 \pm 0,39$ & $0,82 \pm 0,23$ & $1,06 \mathrm{c}$ \\
\hline & S1 & $2,21 \pm 1,15$ & $1,45 \pm 0,35$ & $1,27 \pm 0,50$ & $1,64 \mathrm{~b}$ \\
\hline & S2 & $3,69 \pm 1,44$ & $2,69 \pm 0,74$ & $2,06 \pm 0,59$ & $2,82 \mathrm{a}$ \\
\hline & $\begin{array}{l}\text { Moyenne } \\
\text { provenance }\end{array}$ & $2,38 \mathrm{a}$ & $1,75 \mathrm{~b}$ & $1,39 \mathrm{~b}$ & \\
\hline \multirow[t]{4}{*}{$\mathrm{DT} / \mathrm{SF}$} & S0 & $0,08 \pm 0,03$ & $0,08 \pm 0,02$ & $0,12 \pm 0,02$ & $0,09 \mathrm{c}$ \\
\hline & S1 & $0,19 \pm 0,11$ & $0,12 \pm 0,02$ & $0,19 \pm 0,06$ & $0,17 \mathrm{~b}$ \\
\hline & S2 & $0,65 \pm 0,29$ & $0,5 \pm 0,21$ & $0,42 \pm 0,17$ & $0,53 \mathrm{a}$ \\
\hline & $\begin{array}{l}\text { Moyenne } \\
\text { provenance }\end{array}$ & $0,31 \mathrm{a}$ & $0,23 \mathrm{a}$ & $0,24 \mathrm{a}$ & \\
\hline
\end{tabular}




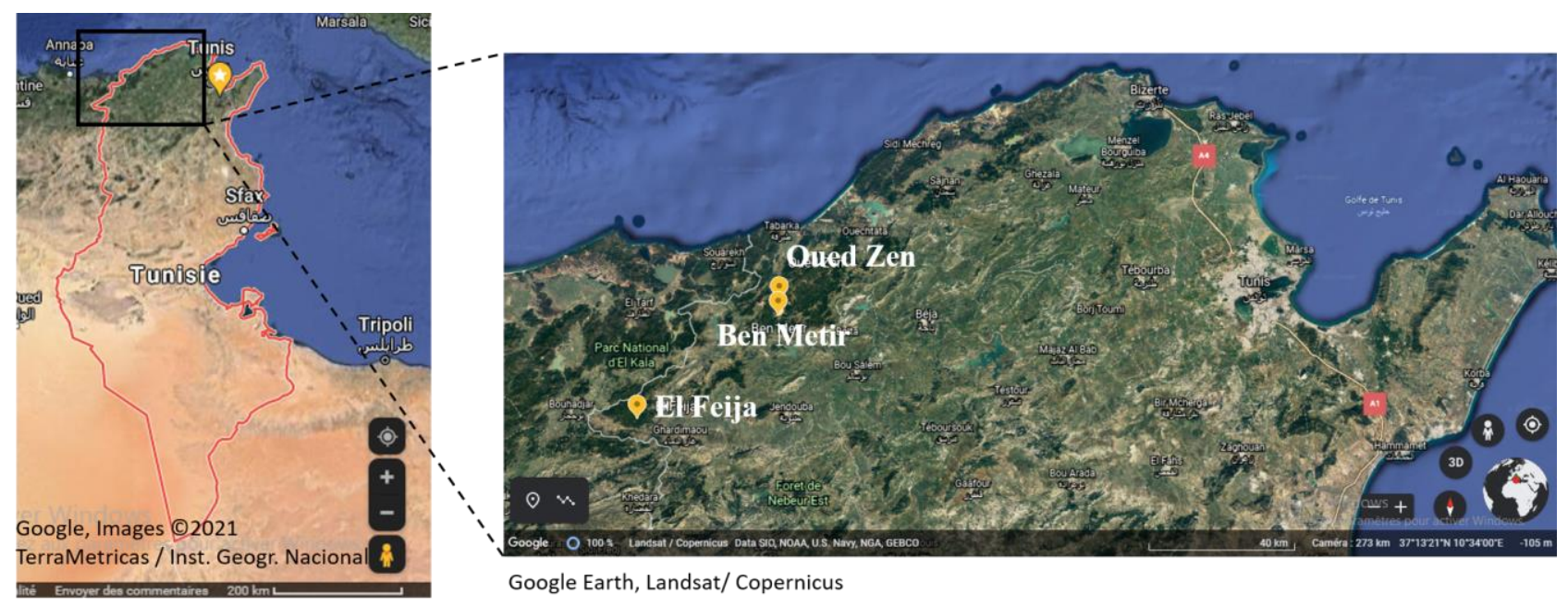

Fig. 1. Localisation des provenances de chêne liège dans la région de la Kroumirie (Nord - Ouest de la Tunisie). 

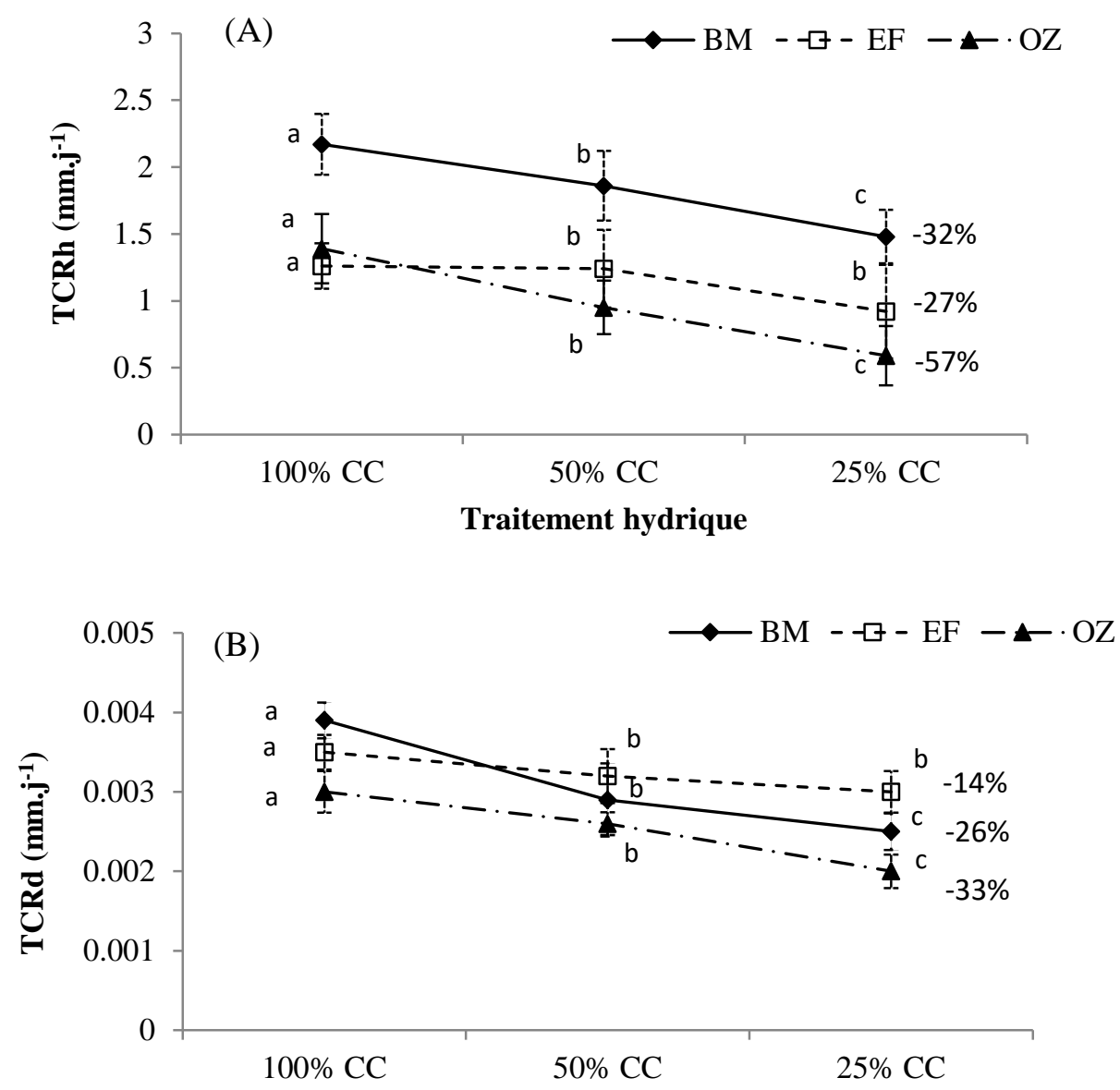

Traitement hydrique

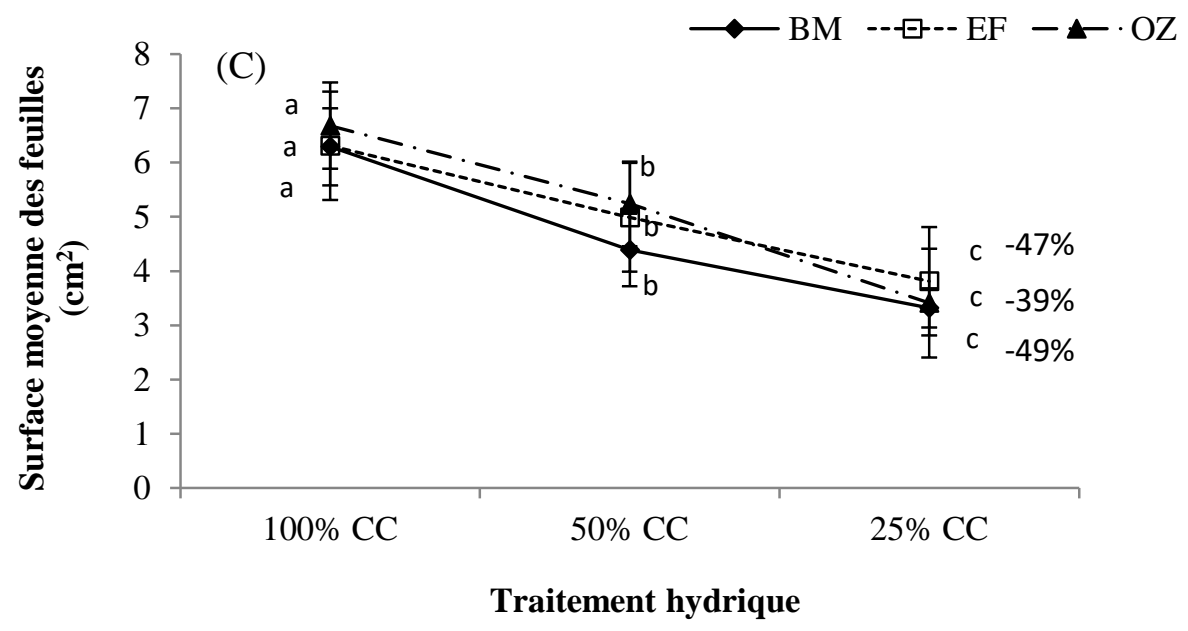

Fig. 2. Taux de croissance relative en hauteur (TCRh) (A), du diamètre au collet (TCRd) (B) et surface moyenne des feuilles (C) chez trois provenances de Quercus suber: Ben Metir $(\mathrm{BM})$, El Feija $(\mathrm{EF})$ et Oued Zen $(\mathrm{OZ})$ soumis à trois traitements hydrique : témoin $(100 \%$ $\mathrm{CC}, \mathrm{S} 0$ ), $50 \% \mathrm{CC}(\mathrm{S} 1)$ et $25 \% \mathrm{CC}(\mathrm{S} 2)$. Pour chaque provenance, les moyennes suivies de la même lettre ne sont pas significativement différentes selon le test de Newman et Keuls ( $\mathrm{p}<$ 0,05).* Taux de réduction du TCRh, TCRd enregistré chez les trois provenances sous $25 \%$ $\mathrm{CC}$. 

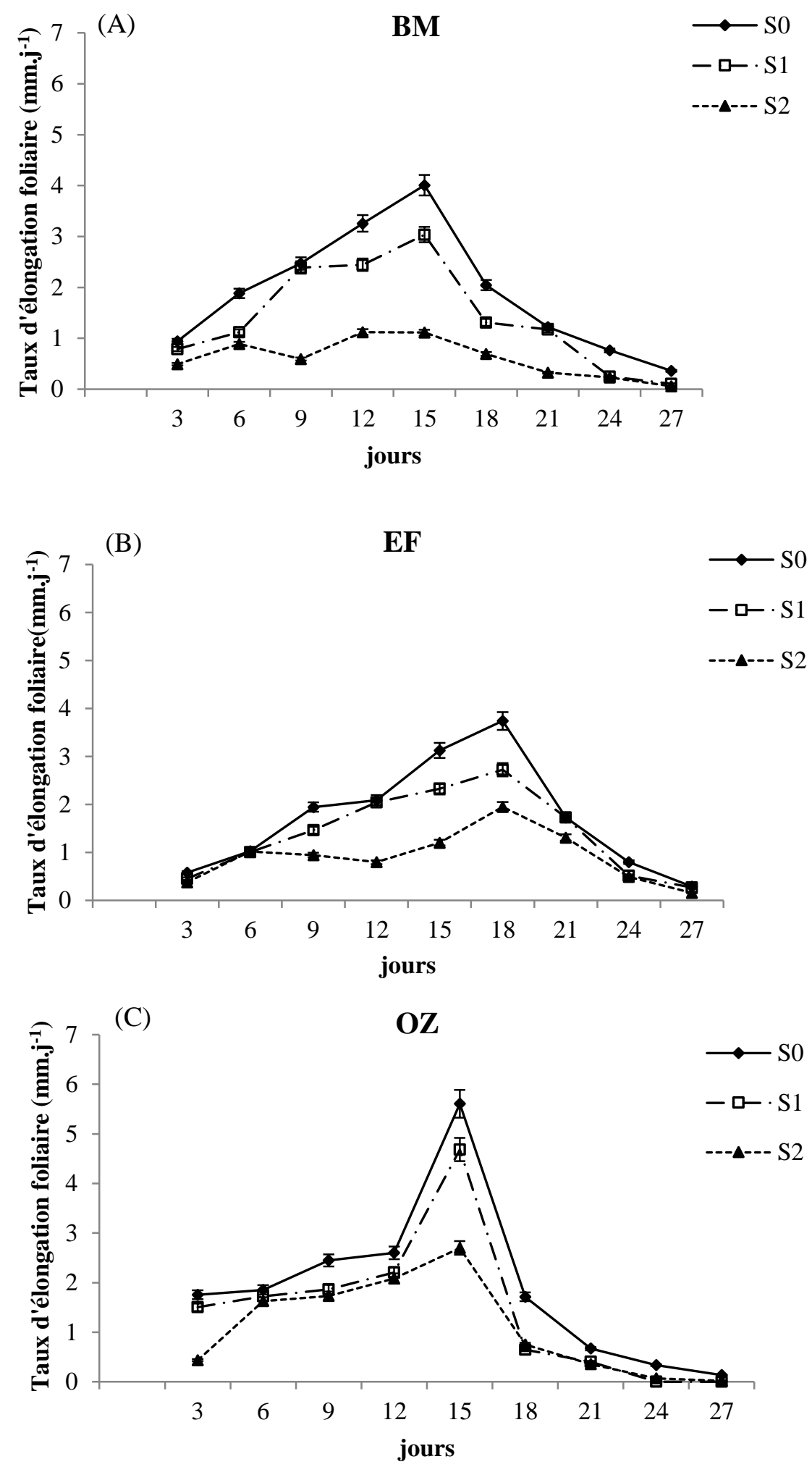

Fig. 3. Taux d'élongation foliaire chez les plants des trois provenances de Quercus suber: Ben Metir (BM) (A), El Feija (EF) (B) et Oued Zen (OZ) (C) soumis à trois traitements hydrique : témoin (100\% CC, S0), 50\% CC (S1) et 25\% CC (S2), n = 12 (moyenne $\pm \mathrm{SD}$ ). 
BM
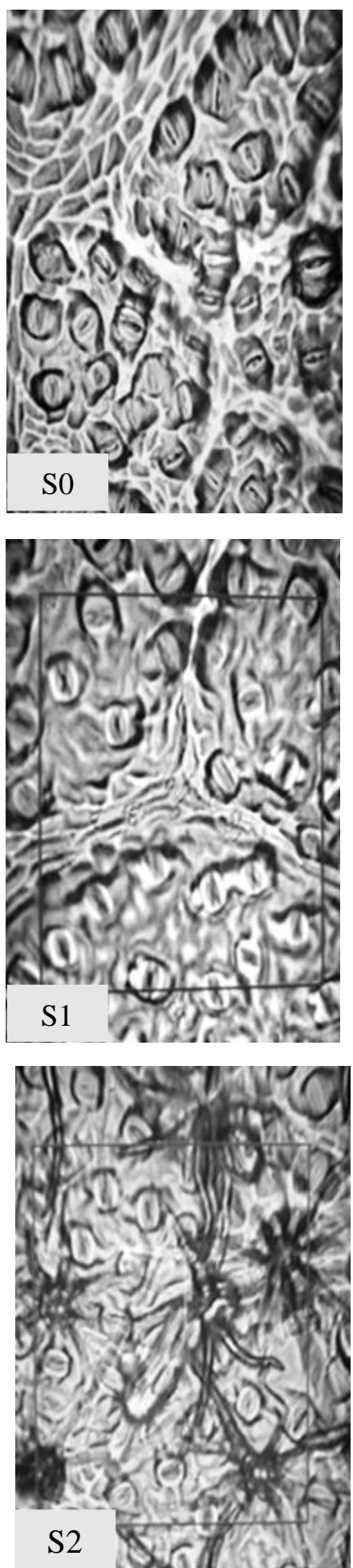

EF
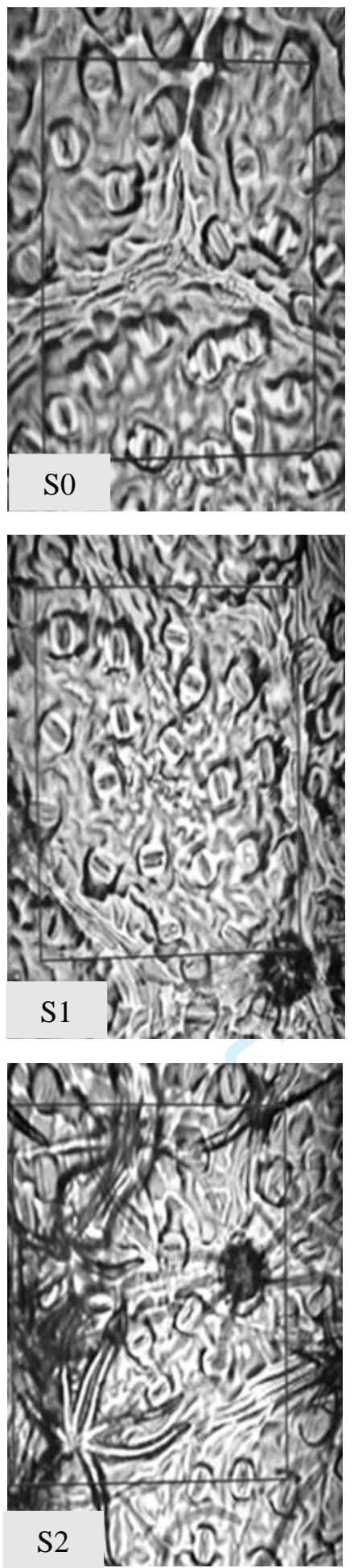

OZ
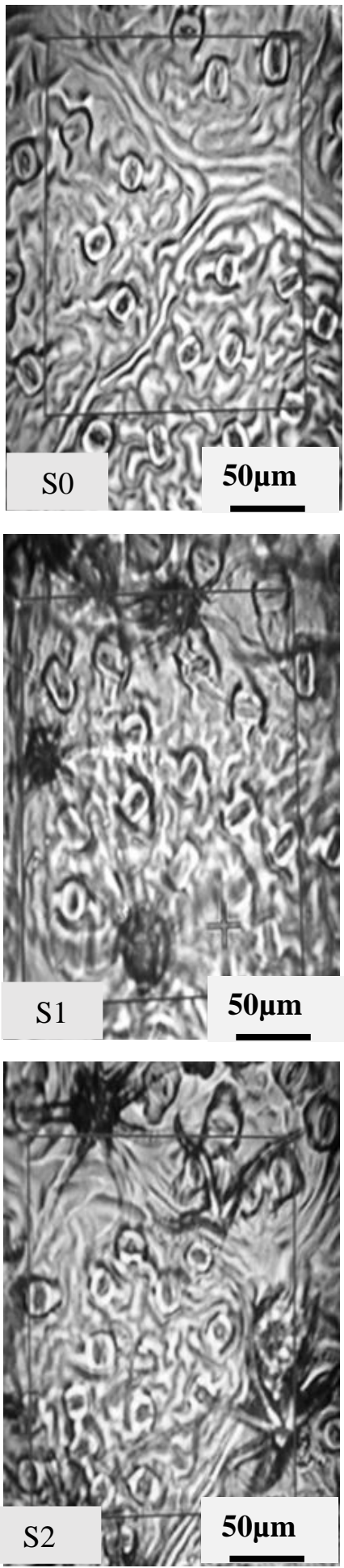

Fig. 4. Observation microscopique des empreintes épidermiques foliaires sur la face abaxiale des feuilles de trois provenances de Quercus suber: Ben Metir (BM), El Feija (EF) et Oued Zen $(\mathrm{OZ})$ soumis à trois traitements hydrique : témoins $(\mathrm{S} 0)$, soumis à $50 \% \mathrm{CC}(\mathrm{S} 1)$ et soumis à $25 \% \mathrm{CC}(\mathrm{S} 2)$ durant 90 jours. L'échelle représente $50 \mu \mathrm{m}$. 


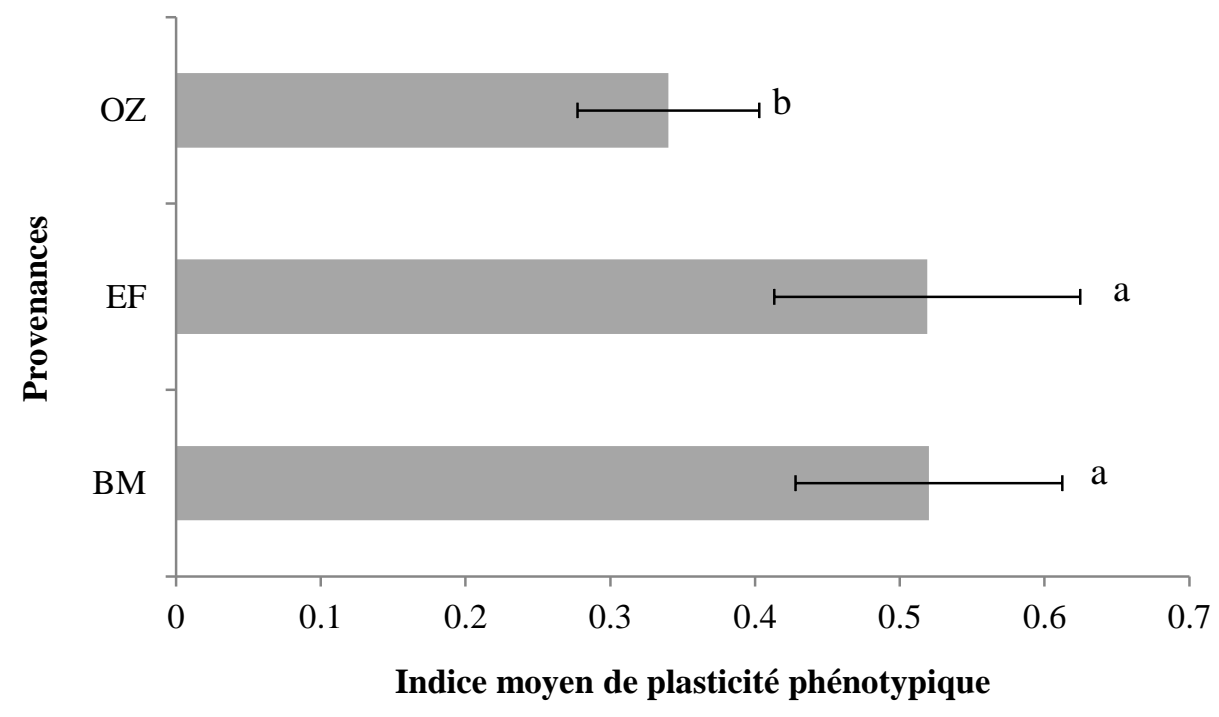

Fig.5. Classification des trois provenances de Quercus suber (Ben Metir (BM), El Feija (EF) et Oued Zen (OZ)) selon l'indice moyen de plasticité phénotypique calculé pour les cinq variables micromorphologiques (densité des stomates, longueur du pore stomatique, densité des trichomes, largeur du trichome et surface relative des trichomes). Les lettres différentes indiquent une différence significative à $\mathrm{p}<0,05$ selon le test de Newman et Keuls entre les provenances. 
(A)
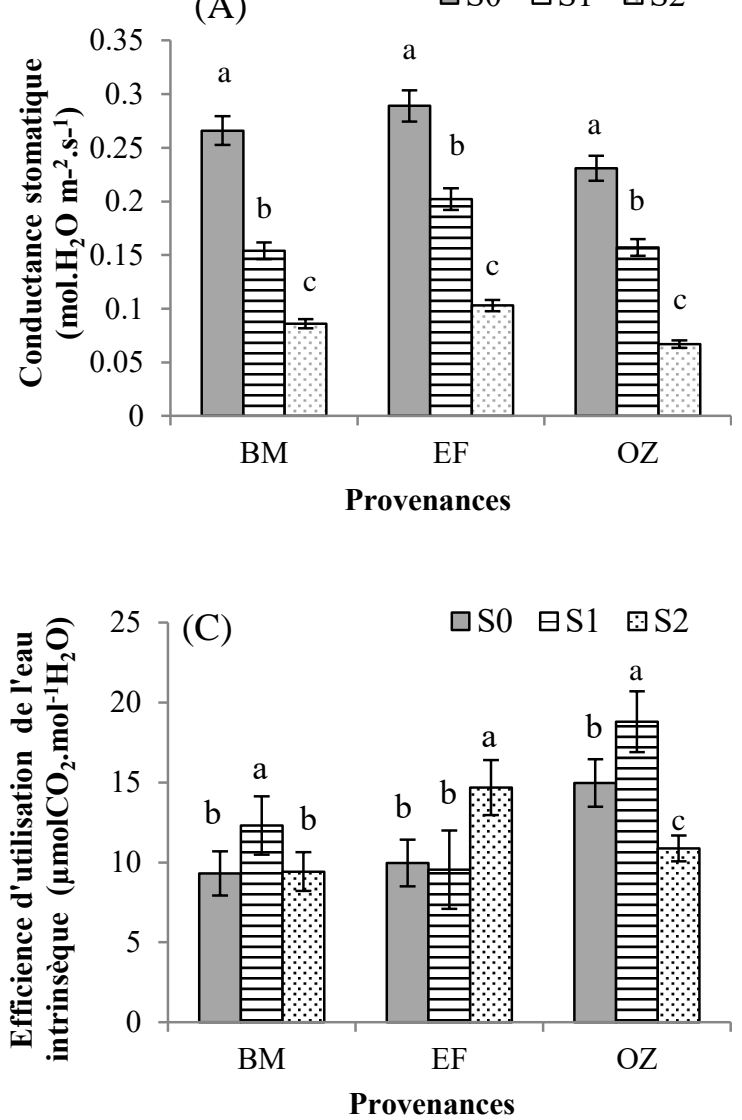

(B)
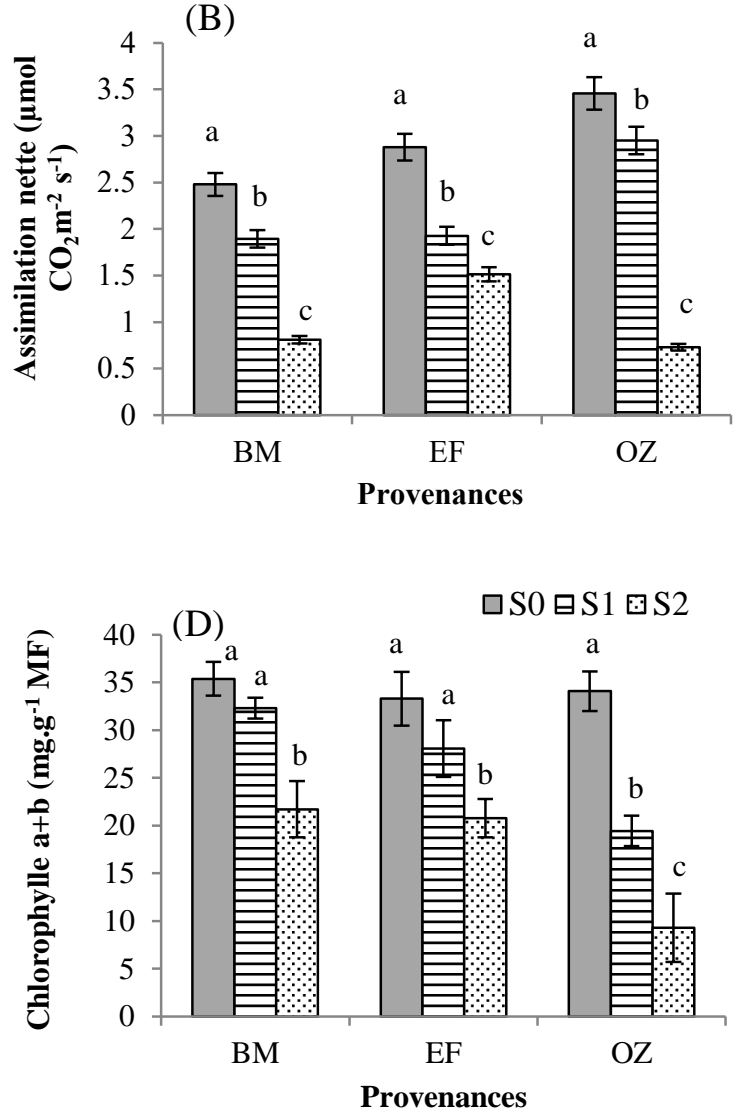

Fig. 6. Effet du stress hydrique sur la conductance stomatique (A), l'assimilation nette (B), l'efficience d'utilisation de l'eau intrinsèque $(C)$ et la concentration en chlorophylles $(a+b)$ (D) chez trois provenances de Quercus suber : Ben Metir (BM), El Feija (EF) et Oued Zen (OZ) soumis à trois traitements hydrique : témoin $(100 \% \mathrm{CC}, \mathrm{S} 0), 50 \% \mathrm{CC}(\mathrm{S} 1)$ et $25 \% \mathrm{CC}$ (S2). Pour chaque provenance, les moyennes au-dessus des barres suivies de la même lettre ne sont pas significativement différentes selon le test de Newman et Keuls $(\mathrm{p}<0,05)$. 